\title{
Dynamic topological logic
}

\author{
Philip Kremer ${ }^{a}, *$, Grigori Mints ${ }^{b}$ \\ ${ }^{a}$ Department of Philosophy, University of Toronto, Canada \\ ${ }^{\mathrm{b}}$ Department of Philosophy, Stanford University, USA
}

Received 4 November 2002; received in revised form 19 March 2004; accepted 3 June 2004

Available online 8 September 2004

Communicated by S. Artemov

\begin{abstract}
Dynamic topological logic provides a context for studying the confluence of the topological semantics for S4, topological dynamics, and temporal logic. The topological semantics for S4 is based on topological spaces rather than Kripke frames. In this semantics, $\square$ is interpreted as topological interior. Thus S4 can be understood as the logic of topological spaces, and $\square$ can be understood as a topological modality. Topological dynamics studies the asymptotic properties of continuous maps on topological spaces. Let a dynamic topological system be a topological space $X$ together with a continuous function $f . f$ can be thought of in temporal terms, moving the points of the topological space from one moment to the next. Dynamic topological logics are the logics of dynamic topological systems, just as S4 is the logic of topological spaces. Dynamic topological logics are defined for a trimodal language with an S4-ish topological modality $\square$ (interior), and two temporal modalities, $\bigcirc$ (next) and $*$ (henceforth), both interpreted using the continuous function $f$. In particular, $\bigcirc$ expresses $f$ 's action on $X$ from one moment to the next, and $*$ expresses the asymptotic behaviour of $f$.

() 2004 Elsevier B.V. All rights reserved.
\end{abstract}

Keywords: Modal logic; Temporal logic; Topological semantics; Topological dynamics

Dynamic Topological Logic (DTL) provides a context for studying the confluence of three research areas: the topological semantics for $\mathrm{S} 4$, topological dynamics, and temporal logic. ${ }^{1}$

\footnotetext{
* Corresponding author.

E-mail addresses: kremer@utsc.utoronto.ca (P. Kremer), mints@csli.stanford.edu (G. Mints).

${ }^{1}$ This study grew out of conversations between Yair Guttman and Grigori Mints, regarding recurrence in measure theory and topological dynamics, and the possibility of expressing this phenomenon in the framework of propositional logic.
}

0168-0072/\$ - see front matter (C 2004 Elsevier B.V. All rights reserved. doi:10.1016/j.apal.2004.06.004 
In the topological semantics for $\mathrm{S} 4$, a model is a topological space $X$ together with a valuation function $V$ assigning to each propositional variable a subset of $X$. Conjunction is interpreted as intersection, disjunction as union, and negation as complementation. If we interpret the necessity connective, $\square$, as topological interior, the resulting modal logic is S4. Thus we can think of S4 as a topological logic, or a logic of topological spaces. ${ }^{2}$

Topological dynamics studies the asymptotic properties of continuous maps on topological spaces ([28, p. 118]). Let a dynamic topological system be an ordered pair $\langle X, f\rangle$ where $X$ is a topological space and $f$ is a continuous function on $X{ }^{3}$ We can think of the function $f$ as moving the points in $X$ in each discrete unit of time: $x$ gets moved to $f x$ and then to $f f x$ and so on. It is natural to extend S4-the logic of (static) topological spaces - to a logic of dynamic topological systems, by adding temporal modalities suited to formalizing the action of $f$ on $X$. In particular, we want to formalize both the transition from one discrete moment to next, as $f$ acts, moment by moment, on the points in $X$; and the asymptotic behaviour of the function $f$.

We turn to $\omega$-time temporal logic with two future-looking modalities: next, $\bigcirc$, and henceforth, *. Suppose that we ignore topological issues and represent discrete moments as natural numbers. We can take an interpretation to be an assignment of a truth value to each propositional variable at each moment. The Boolean connectives are given their standard interpretations. As for the modalities, the formula $\bigcirc A$ is true at the moment $m$ iff $A$ is true at the next moment $m+1$; and the formula $* A$ is true at the moment $m$ iff $A$ is true at the moment $n$, for each $n \geq m$. Note that $* A$ is thus equivalent to the infinite conjunction $A \& \bigcirc A \& \bigcirc^{2} A \& \bigcirc^{3} A$.... We follow [23] in defining the logic W0 to be the set of sentences true at every moment on every interpretation. ${ }^{4}$ W0 can be axiomatized by the classical tautologies; S4 axioms for $* ;(\bigcirc(A \vee B) \equiv(\bigcirc A \vee \bigcirc B)) ;(\bigcirc \neg A \equiv \neg \bigcirc A)$; $(\bigcirc * A \equiv * \bigcirc A) ;(* A \supset \bigcirc A)$; the induction axiom $(A \& *(A \supset \bigcirc A) \supset * A)$; and the rules of modus ponens, and necessitation for $*$.

In this paper, we combine the topological modality and the two temporal modalities, to define trimodal dynamical topological logics: logics of dynamic topological systems. Let a dynamic topological model be an ordered triple $\langle X, f, V\rangle$, where $\langle X, f\rangle$ is a dynamic topological system and $V$ is a valuation function assigning to each propositional variable a subset of $X$. If we think of the subsets of $X$ as the propositions, then, as in the static topological semantics, $\square P=\operatorname{Int}(P)$, for propositions $P$. We interpret the temporal modalities $\bigcirc$ and $*$ using the function $f$. Suppose that, at moment $m$, the proposition $P$ is true at the point $f x$, i.e. $f x \in P$. Then after $f$ has acted on $x$ once, $P$ will be true at $x$. In other words, at the next moment $m+1$, the proposition $P$ is true at the point $x$. So at

\footnotetext{
${ }^{2}$ The topological semantics pre-dates the more well-known Kripke semantics. An interpretation of S4 in the topology of $\mathbb{R}^{2}$ is given, with a soundness proof, in [25]. A general topological semantics is given, with soundness and completeness proofs, in [16]. This work is extended in [17]. For a general and comprehensive discussion, see [20]. See also [1] and [18] for new proofs that S4 is the logic of the closed unit interval.

${ }^{3}$ One might put constraints on $X$, such as being compact or metrizable; and on $f$, such as being bijective, surjective, open or a homeomorphism. Of particular interest to topological dynamicists are measure-preserving functions on compact measure spaces, because of the phenomenon of recurrence. See Section 5, below.

${ }^{4}$ Such a logic was first put forward in [26,27] and [19]. [21] credits Dana Scott, Hans Kamp, and Kit Fine with unpublished axiomatizations and completeness proofs. The first published completeness proof occurs in [22] (a Russian translation of [23], which did not appear in print until 1989). See also [15] and [8].
} 
moment $m$, the proposition $\bigcirc P$ is true at $x$. Thus $x \in \bigcirc P$ iff $f x \in P$ iff $x \in f^{-1}(P)$. Thus our interpretation of the next modality: $\bigcirc P=f^{-1}(P)$. As for $*$, we understand $* P$ as in the preceding paragraph as an infinite conjunction: $P \& \bigcirc P \& \bigcirc^{2} P \& \bigcirc^{3} P \& \ldots$ Thus $* P=\cap_{n \geq 0} f^{-n}(P)$.

Our plan in this paper is as follows. Section 1: we work with a trimodal language, with one topological modality (interior) and two temporal modalities (next and henceforth). We give a precise definition of dynamic topological models-including dynamic Alexandrov models, the dynamic topological analogues of Kripke models-and standard definitions of validity with respect to a model, or a class of models. We give a semantic definition of the dynamic topological logic generated by a class $\mathcal{T}$ of topological spaces and/or a class $\mathcal{F}$ of continuous functions. We also give a precise definition of a fragment of a topological logic. Sections 2-5: we consider various specific DTLs, presenting their properties and axiomatizing some of their next-interior fragments. Section 6: we give conditions under which the purely topological fragment of a DTL is simply S4, and the purely temporal fragment is simply W0. Section 7: we give a sound and complete axiomatization of a DTL in a trimodal fragment of the language in which the temporal modalities cannot occur in the scope of a topological modality. (Nikolai Bjorner originally suggested considering this fragment of the language.)

The current paper is part of a research programme whose first results were announced in three conference abstracts, [11,12], and [13]. (These results are reproduced and proved below.) An independent and closely related research programme saw its first results published in [3], and has been further pursued in [6]. Reference [3] considers two bimodal logics, S4F and S4C: S4C is the next-interior fragment of our basic trimodal logic, generated by the class of all dynamic topological systems; and S4F is the nextinterior fragment of the weaker logic generated by topological structures, i.e. ordered pairs $\langle X, f\rangle$ where $X$ is a topological space and $f$ is a total function, continuous or not, on $X$. (Our dynamic topological systems are their continuous topological structures.) Reference [3] provides both S4F and S4C with Hilbert- and Gentzen-style axiomatizations, cut elimination theorems, both topological and Kripke completeness theorems, and finite model property theorems. We will comment further on [3] as we continue.

Reference [6] continues this work. The richest logic in [6] combines the topological modality with the rich modalities of Propositional Dynamic Logic (PDL), of which the above temporal modalities are a special case. The language of standard PDL has both atomic propositions and atomic actions. The atomic actions are combined to produce composite actions of the form $\alpha \beta, \alpha+\beta$, and $\alpha *$, where $\alpha$ and $\beta$ are actions. With each action $\alpha$ we have a "necessity" modality $[\alpha]$ and a "possibility" modality $\langle\alpha\rangle$, where the formula $[\alpha] A$ reads "action $\alpha$ always makes it the case that $A$ " and the formula $\langle\alpha\rangle A$ reads "action $\alpha$ sometimes makes it the case that $A$ ". The modality $[\alpha *]$ is the result of repeating $[\alpha] \omega$ times, just as the temporal modality $*$ is the result of repeating $\bigcirc$ $\omega$ times. The language in [6] is interpreted via a rich notion of a dynamic topological system: in [6], a topological system, is a topological space $X$ together with a continuous function $f_{\alpha}$ for each atomic action $\alpha$. Reference [6] defines a Hilbert-style axiomatic logic TPDL (Topological Propositional Dynamic Logic), which is sound for the class of all topological systems; and for the class of all topological systems whose underlying topological space is an Alexandrov space (Alexandrov spaces are the topological duals of 
Kripke frames). Reference [6] also claims that TDPL is complete for each of these two classes of topological systems, but this cannot be, since $[\alpha *] \square p \supset \square[\alpha *] p$ is valid when the underlying space is an Alexandrov space, but is not valid in general. (See Section 3 for a proof.) We will comment further on [6] as we continue.

As we were editing the current paper for publication, we received notice of a proof, in [9], of the nonaxiomatizability of a significant range of DTLs: the DTL of homeomorphisms, the DTL of homeomorphisms on $\mathbb{R}^{n}$ (for any fixed $n \geq 1$ ), the DTL of homeomorphisms on Alexandrov spaces (see below), and the DTL of measure-preserving homeomorphisms on the unit ball of dimension $n$, where $n \geq 2$. Reference [9] leaves open the axiomatizability problem of DTLs that are based on continuous functions in general, rather than homeomorphism. We will comment further on [9] as we continue.

\section{Basic definitions}

We work with a trimodal language $L$ with a set $P V$ of propositional variables; Boolean connectives $\vee$ and $\neg$; and three one-place modalities $\square$ (interior), $\bigcirc$ (next), and * (henceforth). We assume that $\&, \supset$, and $\equiv$ are defined in terms of $\vee$ and $\neg$. We will sometimes work with fragments of $L$, but always assume that our fragment contains the Boolean connectives. For example, the $\bigcirc \square$ fragment of $L$, denoted as $L \bigcirc \square$, is the language $L$ without the $*$ modality. We will be particularly interested in the temporalover-topological fragment of $L$, denoted as $L^{\bigcirc * / \square}$ : in this fragment we have all three modalities, but neither $\bigcirc$ nor $*$ can occur in the scope of an occurrence of $\square$. We use $p, q$, $r$ as metavariables over $P V$ and $A, B, C$ as metavariables over formulas.

Definition 1. A topological model is an ordered pair, $M=\langle X, V\rangle$, where $X$ is a topological space and $V: P V \rightarrow \mathcal{P}(X)$. For each formula $B$ in the language $L^{\square}$, we define $M(B)$, the subset assigned by $M$ to $B$ as follows:

$$
\begin{aligned}
& M(p)=V(p), \\
& M(A \vee B)=M(A) \cup M(B), \\
& M(\neg B)=X-M(B), \text { and } \\
& M(\square B)=\operatorname{Int}(M(B)) .
\end{aligned}
$$

We define standard validity relations:

$$
\begin{aligned}
& M \models B \text { iff } M(B)=X . \\
& X \models B \text { iff } M \models B \text { for every model } M=\langle X, V\rangle . \\
& B \text { is valid }(\models B) \text { iff } X \models B \text { for every topological space } X .
\end{aligned}
$$

Definition 2. A Kripke frame is an ordered pair $\langle W, R\rangle$ where $W$ is a non-empty set and $R$ is a reflexive and transitive relation on $W$.

Definition 3. Given a Kripke frame $\langle W, R\rangle$, a subset $S$ of $W$ is open iff $S$ is closed under $R$ : for every $x, y \in W$, if $x \in S$ and $x R y$ then $y \in S$. The family of open sets forms a topology. Thus, for every Kripke frame $\langle W, R\rangle$, we define a dual topological space by 
imposing that topology on the set $W$. Note that, in these spaces, the intersection of arbitrary open sets is open: thus they are Alexandrov spaces, as defined presently.

Definition 4. An Alexandrov space is a topological space in which the intersection of arbitrary open sets is open. Alexandrov spaces were first introduced by [2]; see also [4]. ${ }^{5}$

Definition 5. Given any topological space $X$, define the relation $R_{X}$ on $X$ as follows: $x R_{X} y$ iff $x \in C l\{y\}$, the topological closure of $\{y\} . R_{X}$ is reflexive and transitive, so $\left\langle X, R_{X}\right\rangle$ is a Kripke frame.

Theorem 6. If $X$ is an Alexandrov space, then a subset $Y$ of $X$ is open in $X$ iff $Y$ is open in the Kripke frame $\left\langle X, R_{X}\right\rangle$. Thus, if $X$ is an Alexandrov space, then the topological space that is the dual of the Kripke frame $\left\langle X, R_{X}\right\rangle$ is $X$ itself.

Proof. Suppose that $X$ is an Alexandrov space and $Y \subseteq X$. $(\Rightarrow)$ Suppose that $Y$ is open in $X$. To see that $Y$ is closed under $R_{X}$, suppose that $x \in Y$ and $x R_{X} y$. Then $x \in C l\{y\}$, so every open set containing $x$ also contains $y$. Thus $y \in Y$. $(\Leftarrow)$ Suppose that $Y$ is closed under $R_{X}$. To show that $Y$ is open in the topological space $X$, it suffices to show that the set $Z=X-Y$ is closed. And for this it suffices to show that $C l(Z) \subseteq Z$. Suppose that $z \in C l(Z)$ but that $z \notin Z$. Then $z \in Y$. Let $O_{z}=\cap\{O: O \subseteq X$ and $O$ is open and $z \in O\}$. $O_{z}$ is open since $X$ is an Alexandrov space. So since $z \in \operatorname{cl}(Z)$ there is some $w \in Z \cap O_{z}$. So $w$ is in every open set containing $z$. So $z \in C l\{w\}$. So $z R_{X} w$. So $w \in Y$ since $Y$ is closed under $R_{X}$. But $w \in Z=X-Y$, a contradiction.

Remark 7. Thus Kripke frames are, in effect, Alexandrov spaces, and vice versa.

Remark 8. If $X$ is not an Alexandrov space, then $X$ need not be the topological space dual to the Kripke frame $\left\langle X, R_{X}\right\rangle$. For example, consider the real line $\mathbb{R}$ with the standard topology. Note that the relation $R_{\mathbb{R}}$ is simply the identity relation, $\{\langle x, x\rangle: x \in \mathbb{R}\}$. So in the Kripke frame $\left\langle\mathbb{R}, R_{\mathbb{R}}\right\rangle$, every subset of $\mathbb{R}$ is open. So the topological space that is the dual of $\left\langle\mathbb{R}, R_{\mathbb{R}}\right\rangle$ is not the topological space that we started with: the new topological space is $\mathbb{R}$ with the discrete topology rather than the standard topology.

Definition 9. An Alexandrov model is a topological model $M=\langle X, V\rangle$ where $X$ is an Alexandrov space. This is equivalent to the usual definition of a Kripke model, given the duality of Alexandrov spaces and Kripke frames.

Theorem 10 (McKinsey-Tarski-Kripke). Suppose that $X$ is a dense-in-itself metric space and $A$ is a formula in the language $L^{\square}$. Then the following are equivalent:

(i) $A \in \mathrm{S} 4$.

(ii) $=A$.

(iii) $X \models A$.

\footnotetext{
${ }^{5}$ Alexandrov spaces are the D-topological spaces of [6]. The work in [6] motivated us to discuss Alexandrov spaces.
} 
(iv) $\mathbb{R} \models A$.

(v) $Y \models A$ for every finite topological space $Y$.

(vi) $Y \models A$ for every Alexandrov space $Y$.

Proof. The equivalence of (i)-(v) is due to [17]. For the completeness of S4 in the real line, see the streamlined proofs of [1] and [18]. The equivalence of (i) and (vi) is due, in effect, to [14].

Remark 11. Thus not only does the topological interpretation of $L^{\square}$ give a semantics for S4, but also S4 is the topological logic of a host of particular topological spaces, for example the real line, $\mathbb{R}$; the closed unit interval, $[0,1]$; and any other dense-in-itself metric space. So $L^{\square}$ is expressively weak-unable, for example, to distinguish between $\mathbb{R}$ and $[0,1]$ despite their topological dissimilarities.

Definition 12. A dynamic topological system (DTS) is an ordered pair, $\langle X, f\rangle$, where $X$ is a topological space and $f$ is a continuous function on $X$. (This terminology is adapted from [5] and [7].) A dynamic topological model (DTM) is an ordered triple $M=\langle X, f, V\rangle$ where $\langle X, f\rangle$ is a DTS and $V$ assigns a subset of $X$ to each $p \in P V$. For each formula $B$ we define $M(B)$, the subset assigned by $M$ to $B$, by the clauses in Definition 1.1 plus the following:

$$
\begin{aligned}
& M(\bigcirc B)=f^{-1}(B) ; \text { and } \\
& M(* B)=\cap_{n \geq 0} f^{-n}(B) .
\end{aligned}
$$

Definition 13. A dynamic Alexandrov system is an ordered pair $\langle X, f\rangle$ where $X$ is an Alexandrov space and $f$ is a continuous function on $X$. The continuity of $f$ is equivalent to its monotonicity in the following sense: if $x R_{X} y$ then $(f x) R_{X}(f y) .{ }^{6}$ An dynamic Alexandrov model is a DTM $\langle X, f, V\rangle$ where $X$ is an Alexandrov space.

Definition 14. Suppose that $M=\langle X, f, V\rangle$ is a DTM. We define standard validity relations:

$$
\begin{aligned}
& M \models B \text { iff } M(B)=X . \\
& \langle X, f\rangle \models B \text { iff } M \models B \text { for every model } M=\langle X, f, V\rangle . \\
& X \models B \text { iff }\langle X, f\rangle \models B \text { for every continuous function } f . \\
& B \text { is valid }(\models B) \text { iff } X \models B \text { for every topological space } X .
\end{aligned}
$$

Definition 15. Suppose that $\mathcal{F}$ is a class of functions so that each $f \in \mathcal{F}$ is a continuous function on some topological space. Suppose that $\mathcal{T}$ is a class of topological spaces. We define three more validity relations:

$\mathcal{T}, \mathcal{F} \models B$ iff, for every $f \in \mathcal{F}$ and every $X \in \mathcal{T}$, if $f$ is a continuous function on $X$ then $\langle X, f\rangle \models B$.

\footnotetext{
${ }^{6}$ The monotonicity condition characterizes the continuous Kripke frames of [3].
} 
$\mathcal{F} \models B$ iff, for every topological space $X$ and every $f \in \mathcal{F}$, if $f$ is a continuous function on $X$ then $\langle X, f\rangle \models B$.

$\mathcal{T} \models B$ iff $X \models B$ for every topological space $X \in \mathcal{T}$.

Here we assume that in specifying a particular continuous function, we specify both the function itself as a set of ordered pairs, and the topological space on which we are taking it to act.

We are now ready to define various Dynamic Topological Logics, or DTLs.

Definition 16. For any class $\mathcal{T}$ of topological spaces and any class $\mathcal{F}$ of continuous functions, we define

$$
\begin{aligned}
& \operatorname{DTL}_{\mathcal{T}, \mathcal{F}}=\{A: \mathcal{T}, \mathcal{F} \models A\} . \\
& \operatorname{DTL}_{\mathcal{T}}=\{A: \mathcal{T} \models A\} . \\
& \operatorname{DTL}_{\mathcal{F}}=\{A: \mathcal{F} \models A\} .
\end{aligned}
$$

Given a particular DTL, we will also be interested in its fragments.

Definition 17. If $D$ is a dynamic topological logic, then the purely topological fragment of $D$ is the fragment expressible in the language $L^{\square}$, that is, the set of formulas in $L^{\square}$ belonging to $D$. The purely temporal fragment of $D$ is the fragment expressible in the language $L^{\bigcirc *}$. The next-interior fragment of $D$ is the fragment expressible in the language $L \bigcirc \square$. We denote these logics as $D^{\square}$ and $D^{\bigcirc *}$ and $D^{\bigcirc \square}$, respectively. The temporal-overtopological fragment of $D$ is the fragment expressible in the language $L \bigcirc * / \square$. We denote this logic as $D^{\mathrm{*} / \square}$.

Our research plan is to consider the properties of various DTLs and their fragments, particularly those determined by interesting classes of topological spaces or continuous functions or both. The next four sections specify four DTLs: the DTL of all dynamic topological systems, $\mathrm{DTL}_{0}$; the DTL of Alexandrov spaces, $\mathrm{DTL}_{\mathcal{A}}$; the DTL of homeomorphisms, DTL $\mathcal{H}$; and the DTL of measure-preserving functions on the closed unit interval, DTL $\mathrm{M}_{\mathcal{M}}$. The second of these in nonaxiomatizable ([9]), and the question of the axiomatizability of the other three is still open. Below, we axiomatize some interesting fragments. We also begin the process of investigating the expressive resources of the trimodal language $L$ and its fragments by comparing various DTLs and their fragments. Along these lines, we hope eventually to prove or disprove analogues to the McKinseyTarski-Kripke Theorem 10, above.

\section{Basic DTL}

Our most basic DTL is the following:

$$
\mathrm{DTL}_{0}=\{A: \models A\} .
$$

It is not known whether $\mathrm{DTL}_{0}$ is axiomatizable. In this section, we give [3]'s axiomatization of its next-interior fragment, and in Section 7 we axiomatize its 
temporal-over-topological fragment. [3]'s logic S4C in the language $L^{\bigcirc} \square$ is given by the following axioms,

$$
\begin{aligned}
& \text { the classical tautologies, } \\
& \text { S4 axioms for } \square \text {, } \\
& (\bigcirc(A \vee B) \equiv(\bigcirc A \vee \bigcirc B) \text { ), } \\
& (\bigcirc \neg A \equiv \neg \bigcirc A) \text {, and } \\
& (\bigcirc \square A \supset \square \bigcirc A \text { ) (the axiom of continuity); }
\end{aligned}
$$

and the rules of modus ponens, and necessitation for both $\bigcirc$ and $\square$. We will use S4C both for this axiomatization and for the set of all formulas derivable from the axioms by the inference rules. As it turns out, this axiomatization is both sound and complete with respect to the class of all dynamic topological models, as well as the class of all dynamic Alexandrov models (see Definition 13). Soundness is easy to establish: in our terminology, soundness amounts to the claim that $\mathrm{S} 4 \mathrm{C} \subseteq \mathrm{DTL}_{0}^{\bigcirc \square}$ and $\mathrm{S} 4 \mathrm{C} \subseteq \mathrm{DTL}_{\mathcal{A}}^{\bigcirc}$. [3] prove completeness with respect to both classes of models-in our terminology, $\mathrm{DTL}_{0}^{\bigcirc \square} \subseteq \mathrm{S} 4 \mathrm{C}$ and DTL ${ }_{\mathcal{A}}^{\bigcirc \square} \subseteq \mathrm{S} 4 \mathrm{C}$ - as well as the finite model property. [6] gives a simpler proof of the completeness result; we reproduce that proof here.

First we define some standard notions: $A$ is a theorem iff $A \in \mathrm{S} 4 \mathrm{C}$. $A$ is consistent iff $\neg A \notin \mathrm{S} 4 \mathrm{C}$. A theory is a set of formulas in the language $L \bigcirc \square$ containing all the theorems of S4C and closed under modus ponens. A theory $T$ is complete iff for every formula $A$ either $A \in T$ or $\neg A \in T$. A theory $T$ is consistent iff some formula is not in $T$. A set $S$ of formulas is consistent iff some theory $T \supseteq S$ is consistent.

Theorem 18 ([3] and [6]). $\mathrm{S} 4 \mathrm{C}=\mathrm{DTL}_{0}^{\bigcirc \square}=\mathrm{DTL}_{\mathcal{A}}^{\bigcirc \square}$.

Proof. Given soundness and the obvious fact that $\mathrm{DTL}_{0}^{\bigcirc \square} \subseteq \mathrm{DTL}_{\mathcal{A}}^{\bigcirc \square}$, it suffices to construct a canonical dynamic Alexandrov model $M$ (see Definition 13) such that $M \models A$ iff $A \in \mathrm{S} 4 \mathrm{C}$, for every formula $A$ in the language $L \bigcirc \square$. In fact, given soundness, it will suffice to show that if $M=A$ then $A \in \mathrm{S} 4 \mathrm{C}$.

Define a Kripke frame $\langle X, R\rangle$ and a function $f$ on $X$ as follows:

$$
\begin{aligned}
& X=\{x: x \text { is a complete consistent theory }\} \\
& x R y \text { iff for every formula } A, \text { if } \square A \in x \text { then } A \in y ; \text { and } \\
& f x=\{A: \bigcirc A \in x\} .
\end{aligned}
$$

Note that $R$ is reflexive since $(\square A \supset A) \in \mathrm{S} 4 \mathrm{C}$ and transitive since $(\square A \supset \square \square A) \in$ $\mathrm{S} 4 \mathrm{C}$. We can take $X$ to be an Alexandrov space, as in Definition 2, by imposing the following topology on it: a subset $Y$ of $X$ is open iff $Y$ is closed under $R$ : for every $x, y \in X$, if $x R y$ and $x \in Y$ then $y \in Y$.

Now we show that $f$ is continuous. It suffices to show that $f$ is monotone in the following sense: $x R y \Rightarrow(f x) R(f y)$. So suppose that $x R y$. To see that $(f x) R(f y)$, suppose $\square A \in f x$. Then $\bigcirc \square A \in x$. So $\square \bigcirc A \in x$, since $(\bigcirc \square A \supset \square \bigcirc A) \in \mathrm{S} 4 \mathrm{C}$. So $\bigcirc A \in y$. So $A \in f y$, as desired. 
Thus $\langle X, f\rangle$ is a dynamic Alexandrov system. Define $V(p)=\{x \in X: p \in x\}$. Then $M=\langle X, f, V\rangle$ is a dynamic Alexandrov model. By a standard induction on the complexity of the formula $A$, we have $x \in M(A)$ iff $A \in x$, for every $x \in X$.

To show that if $M \models A$ then $A \in \mathrm{S} 4 \mathrm{C}$, suppose that $A \notin \mathrm{S} 4 \mathrm{C}$. Then $\neg A$ is consistent. By a standard argument, every consistent formula is a member of some complete consistent theory. So $\neg A \notin x$, for some $x \in X$. So $x \notin M(A)$. So $M \not \models A$, as desired.

Theorem 18 suggests the following conjecture:

Conjecture 19. $\mathrm{DTL}_{0}$ can be axiomatized, in the trimodal language, by combining the axioms of $\mathrm{W} 0$ and $\mathrm{S} 4 \mathrm{C}$, with the rules of modus ponens and necessitation for all three modalities.

Theorem 18 is partially analogous to the McKinsey-Tarski-Kripke Theorem 10 for the language $L^{\bigcirc \square}$ : for every formula $A$ of $L \bigcirc \square$, we have $A \in \mathrm{S} 4 \mathrm{C}$ iff $\models A$ iff $Y \models A$ for every Alexandrov space $Y$. The next theorem, however, reveals a disanalogy: it is not the case that $A \in \mathrm{S} 4 \mathrm{C}$ iff $\mathbb{R} \models A$.

Theorem 20. $\mathrm{DTL}_{0}^{\bigcirc \square} \subsetneq \mathrm{DTL}_{\mathbb{R}}^{\bigcirc \square}$, and $\mathrm{DTL}_{0} \subsetneq \mathrm{DTL}_{\mathbb{R}}$.

Proof. Clearly $\mathrm{DTL}_{0}^{\bigcirc \square} \subseteq \mathrm{DTL}_{\mathbb{R}}^{\bigcirc \square}$, and $\mathrm{DTL}_{0} \subseteq \mathrm{DTL}_{\mathbb{R}}$. So it suffice to show that $\mathrm{DTL}_{\mathbb{R}}^{\bigcirc \square} \nsubseteq \mathrm{DTL}_{0}^{\bigcirc \square}$. Consider the following formula $A$, where $p$ and $q$ are propositional variables:

$(\square \bigcirc p \supset \bigcirc \diamond \square p) \vee(\bigcirc q \supset \square \bigcirc q)$.

We will show that $A \in \mathrm{DTL}_{\mathbb{R}}^{\bigcirc \square}-\mathrm{DTL}_{0}^{\bigcirc \square}$.

We first show that $A \notin \mathrm{DTL}_{0}^{\bigcirc \square}$. Let $M=\langle X, f, V\rangle$, where

$$
X=\{0,1,2\}
$$

the open sets are $\emptyset, X$, and $\{2\}$;

$f(2)=f(1)=0$ and $f(0)=1$; and

$V(p)=\{0,1\}$, and $V(q)=\{1\}$.

Note the following:

$$
\begin{aligned}
& V(\bigcirc p)=X ; \text { so } V(\square \bigcirc p)=X . \\
& V(\square p)=\emptyset ; \text { so } V(\bigcirc \diamond \square p)=\emptyset .
\end{aligned}
$$

Thus $V(\square \bigcirc p \supset \bigcirc \diamond \square p)=\emptyset$.

Meanwhile, $V(\bigcirc q)=\{0\}$; so $V(\square \bigcirc q)=\emptyset$.

Thus $V(\bigcirc q \supset \square \bigcirc q)=\{1,2\}$.

Thus $V(A)=\{1,2\} \neq X$.

Thus $M \not \models A$. 
We now show that $A \in \mathrm{DTL}_{\mathbb{R}}^{\bigcirc \square}$. Suppose not. Then there is some dynamic topological model $M^{\prime}=\left\langle\mathbb{R}, f^{\prime}, V^{\prime}\right\rangle$ and some $x \in \mathbb{R}$ such that $x \notin V^{\prime}(A)$. Thus,

(i) $x \in V^{\prime}(\square \bigcirc p)$. So there is an open interval $I$ such that $x \in I \subseteq V^{\prime}(\bigcirc p)$. So $f^{\prime}(x) \in f^{\prime}(I) \subseteq V^{\prime}(p)$

(ii) $x \notin V^{\prime}(\bigcirc \diamond \square p)$. So $f^{\prime}(x) \notin C l\left(\operatorname{Int}\left(V^{\prime}(p)\right)\right)$.

(iii) $x \in V^{\prime}(\bigcirc q)$. So $f^{\prime}(x) \in V(q)$.

(iv) $x \notin V^{\prime}(\square \bigcirc q)$. So there is some $y \in I$ such that $y \notin V^{\prime}(\bigcirc q)$. Thus $f^{\prime}(y) \notin V^{\prime}(q)$. Thus $f^{\prime}(x) \neq f^{\prime}(y)$. Thus $f^{\prime}(I)$ is not a singleton set.

Since $f^{\prime}(I)$ is not a singleton set and since $I$ is an open interval, $f^{\prime}(I)$ is either an open interval, a closed interval, or a semi-closed interval, i.e. an interval of the form $[a, b)$ or $(a, b]$. In any case, $f^{\prime}(I) \subseteq C l\left(\operatorname{Int}\left(f^{\prime}(I)\right)\right)$. And since from (i) we have $f^{\prime}(I) \subseteq V^{\prime}(p)$, we also have

$$
f^{\prime}(x) \in f^{\prime}(I) \subseteq C l\left(\operatorname{Int}\left(f^{\prime}(I)\right)\right) \subseteq C l\left(\operatorname{Int}\left(V^{\prime}(p)\right)\right) .
$$

But this contradicts (ii).

Remark 21. Theorem 20 was discovered independently by [24], with a different counterexample.

Conjecture 22. $\mathrm{DTL}_{\mathbb{R}}^{\mathrm{O}}=\mathrm{S} 4 \mathrm{C}+((\square \bigcirc A \supset \bigcirc \diamond \square A) \vee(\bigcirc B \supset \square \bigcirc B))$.

\section{The DTL of Alexandrov spaces}

Of particular interest is the class $\mathcal{A}$ of dynamic Alexandrov models (see Definition 13), since these are the models based on Alexandrov spaces, which are, in effect, Kripke frames (see Remark 7). The fact that $\mathrm{DTL}_{0} \subsetneq \mathrm{DTL}_{\mathcal{A}}$ follows from $(*)$ and $(\dagger)$, below:

$$
\begin{aligned}
& (* \square p \supset \square * p) \notin \mathrm{DTL}_{0} \\
& (* \square p \supset \square * p) \in \mathrm{DTL}_{\mathcal{A}} .
\end{aligned}
$$

$(\dagger)$ follows from the fact that, in an Alexandrov space, the intersection of arbitrary open sets is open. To see $(*)$, let $M=\langle\mathbb{R}, f, V\rangle$ where $f(x)=2 x$ and $V(p)=(-1,1)$. Note that $M(\square p)=(-1,1)$, so $f^{-n}(M(\square p))=\left(-1 / 2^{n}, 1 / 2^{n}\right)$. Thus $M(* \square p)=\{0\}$. Similarly, $M(* p)=\{0\}$. So $M(\square * p)=\emptyset$. So $M \not \models(* \square p \supset \square * p)$.

By Theorem 18, above, DTL ${ }_{\mathcal{A}}^{\bigcirc}=\mathrm{DTL}_{0}^{\bigcirc \square}=\mathrm{S} 4 \mathrm{C}$. And by Corollary 44, below, $\mathrm{DTL}_{\mathcal{A}}{ }^{*}=\mathrm{DTL}_{0}^{\bigcirc *}=\mathrm{W} 0$. So any differences between $\mathrm{DTL}_{\mathcal{A}}$ and $\mathrm{DTL}_{0}$ should arise from the interaction of $*$ and $\square$ :

Conjecture 23. $\mathrm{DTL}_{\mathcal{A}}=\mathrm{DTL}_{0}+(* \square p \supset \square * p)$.

Remark 24. We do not know whether $\mathrm{DTL}_{\mathcal{A}}$ is axiomatizable.

\section{The DTL of homeomorphisms}

Of particular interest is the class $\mathcal{H}$ of homeomorphisms (continuous bijections with continuous inverses). Intuitively, we keep track of time with $f$. Although our temporal 
modalities are forward-looking, it seems natural to keep track of time with functions that can look in both directions (i.e. that are bijective) and that are continuous in both directions. Despite the fact that our temporal modalities are forward-looking, restricting our attention to the class $\mathcal{H}$ makes a difference that can be expressed in our trimodal propositional language. In particular we have $(*)$ and $(\dagger)$, below:

$$
\begin{aligned}
& (\square \bigcirc p \supset \bigcirc \square p) \notin \text { DTL }_{0} . \\
& (\square \bigcirc p \supset \bigcirc \square p) \in \text { DTL }_{\mathcal{H}} .
\end{aligned}
$$

$(\dagger)$ follows from the fact that $\operatorname{Int}\left(f^{-1}(S)\right) \subseteq f^{-1}(\operatorname{Int}(S))$ where $S$ is a subset of a topological space $X$ on which $f$ is a homeomorphism. To see (*), let $M=\langle X, f, V\rangle$ where $X=\{0,1\}$ with open sets $\emptyset,\{0\}$, and $\{0,1\}$; and where $f(0)=f(1)=1$ and $V(p)=\{1\}$. The function $f$ is continuous and hence $M$ is a DTM. Also note that $M(\square \bigcirc p)=\{0,1\}$ and $M(\bigcirc \square p)=\emptyset$, so $M \not \forall(\square \bigcirc p \supset \bigcirc \square p)^{7}$

As mentioned above, [9] presents a proof that DTL $\mathrm{H}_{\mathcal{H}}$ is not axiomatizable. We do, however, have an axiomatization of its next-interior fragment. Define the logic S4O in the language $L^{\bigcirc}$ by adding the following axiom to S4C, defined in Section 2, above:

\section{$(\square \bigcirc A \supset \bigcirc \square A)$.}

It turns out that $\mathrm{S} 4 \bigcirc=\mathrm{DTL}_{\mathcal{H}}^{\bigcirc \square}$. What is more, Theorem 25 extends this to give an analogue to the McKinsey-Tarski-Kripke Theorem 10:

Theorem 25. $\mathrm{S} 4 \bigcirc=\mathrm{DTL}_{\mathcal{H}}^{\bigcirc \square}=\mathrm{DTL}_{\mathbb{R}, \mathcal{H}}^{\bigcirc \square}=\mathrm{DTL}_{[0,1], \mathcal{H}}^{\bigcirc \square}=\mathrm{DTL}_{\mathcal{A}, \mathcal{H}}^{\bigcirc \square}=\mathrm{DTL}_{\mathcal{O}}^{\bigcirc \square}=$ $\mathrm{DTL}_{\mathbb{R}, \mathcal{O}}^{\bigcirc \square}=\mathrm{DTL}_{[0,1], \mathcal{O}}^{\bigcirc \square}=\mathrm{DTL}_{\mathcal{A}, \mathcal{O}}^{\bigcirc}$

Proof. ${ }^{8}$ The claim that $\mathrm{S} 4 \bigcirc \subseteq \mathrm{DTL}_{\mathcal{O}} \mathrm{O}$ is just a version of soundness, which is proved as usual. Given this, the following inclusion relations are obvious:

$$
\begin{aligned}
& \mathrm{S} 4 \bigcirc \subseteq \mathrm{DTL}_{\mathcal{O}}^{\bigcirc \square} \subseteq \mathrm{DTL}_{\mathcal{H}}^{\bigcirc \square} \subseteq \mathrm{DTL}_{\mathbb{R}, \mathcal{H}}^{\bigcirc \square} \\
& \mathrm{S} 4 \bigcirc \subseteq \mathrm{DTL}_{\mathcal{O}}^{\bigcirc \square} \subseteq \mathrm{DTL}_{\mathbb{R}, \mathcal{O}}^{\bigcirc \square} \subseteq \mathrm{DTL}_{\mathbb{R}, \mathcal{H}}^{\bigcirc \square} \\
& \mathrm{S} 4 \bigcirc \subseteq \mathrm{DTL}_{\mathcal{O}}^{\bigcirc \square} \subseteq \mathrm{DTL}_{\mathcal{H}}^{\bigcirc \square} \subseteq \mathrm{DTL}_{[0,1], \mathcal{H}}^{\bigcirc \square} \\
& \mathrm{S} 4 \bigcirc \subseteq \mathrm{DTL}_{\mathcal{O}}^{\bigcirc \square} \subseteq \mathrm{DTL}_{[0,1], \mathcal{O}}^{\bigcirc \square} \subseteq \mathrm{DTL}_{[0,1], \mathcal{H}}^{\bigcirc \square} \\
& \mathrm{S} 4 \bigcirc \subseteq \mathrm{DTL}_{\mathcal{O}}^{\bigcirc \square} \subseteq \mathrm{DTL}_{\mathcal{A}, \mathcal{O}}^{\bigcirc \square} \subseteq \mathrm{DTL}_{\mathcal{A}, \mathcal{H}}^{\bigcirc \square} .
\end{aligned}
$$

\footnotetext{
${ }^{7}$ [3] notes that the axiom scheme $(\square \bigcirc A \supset \bigcirc \square A$ ) characterizes topological structures with open functions, i.e. functions that map open sets to open sets; and that this axiom scheme together with $(\bigcirc \square A \supset \square \bigcirc A$ ) characterizes topological structures with continuous and open functions. Theorem 25, below, strengthens this, by showing, in effect, that these two axiom schemes not only characterize the dynamic topological systems whose functions are continuous and open, but also the dynamic topological systems whose functions are homeomorphisms, i.e. continuous and open bijections.

8 Vladimir Rybakov helped us with this proof.
} 
So it suffices to show, for every formula $A$ in the language $L \bigcirc \square$ :

$$
\begin{aligned}
& \text { if }[0,1], \mathcal{H} \models A \text { then } \mathbb{R}, \mathcal{H} \models A, \\
& \text { if } \mathbb{R}, \mathcal{H} \models A \text { then } A \in \mathrm{S} 4 \bigcirc, \text { and } \\
& \text { if } \mathcal{A}, \mathcal{H} \models A \text { then } A \in \mathrm{S} 4 \bigcirc .
\end{aligned}
$$

See Theorems 26, 33 and 34, respectively.

Theorem 26. If $[0,1], \mathcal{H} \models A$ then $\mathbb{R}, \mathcal{H} \models A$.

Proof. Suppose that $\mathbb{R}, \mathcal{H} \not A$. Let $M=\langle\mathbb{R}, f, V\rangle$ be a model where $f$ is a homeomorphism on $\mathbb{R}$ and where $M \not \models A$. Since $f$ is a homeomorphism on $\mathbb{R}, f$ is either strictly increasing or strictly decreasing. (In fact, as we show in the proof of Theorem 33, we can take $f$ to be $f(x)=x+1$. But we will continue with the more general case for now, since we have not yet shown Theorem 33.) Choose some strictly increasing continuous one-one function $h$ from $\mathbb{R}$ onto the open interval $(0,1)$. Define $f^{\prime}$ on $[0,1]$ as follows:

$$
\begin{aligned}
& f^{\prime}(x)=h f h^{-1}(x) \text { if } 0<x<1 ; \\
& f^{\prime}(x)=x \text { if } f \text { is strictly increasing and either } x=0 \text { or } x=1 ; \\
& f^{\prime}(x)=1-x \text { if } f \text { is strictly decreasing and either } x=0 \text { or } x=1 .
\end{aligned}
$$

And define $V^{\prime}(p)=\left\{x \in(0,1): h^{-1}(x) \in V(p)\right\}$.

$f^{\prime}$ is one-one and onto. $f^{\prime}$ is also continuous. For if $f$ is strictly increasing then $\lim _{x \rightarrow 0} f^{\prime}(x)=0$ and $\lim _{x \rightarrow 1} f^{\prime}(x)=1$; and if $f$ is strictly decreasing then $\lim _{x \rightarrow 0} f^{\prime}(x)=1$ and $\lim _{x \rightarrow 1} f^{\prime}(x)=0$. So $M^{\prime}=\left\langle[0,1], f^{\prime}, V^{\prime}\right\rangle$ is a dynamic topological model.

Notice that $(0,1) \cap M^{\prime}(B)=\left\{x \in(0,1): h^{-1}(x) \in M(B)\right\}$, for every formula $B$. The proof of this is a routine induction on formulas. So $M^{\prime}(A) \neq[0,1]$. For otherwise we would have $M(A)=\mathbb{R}$, which is false. So $[0,1], \mathcal{H} \not \forall A$, as desired.

Before we prove Theorems 33 and 34, we give some definitions and lemmas.

Definition 27. Given a formula $B$, let $g(B)$ be the result of pushing all the occurrences of $\bigcirc$ to the atomic formulas. For example, $g(\bigcirc(\bigcirc \square(p \vee \bigcirc q) \vee \bigcirc \neg r))=(\square(\bigcirc \bigcirc p \vee$ $\bigcirc \bigcirc \bigcirc q) \vee \neg \bigcirc \bigcirc r)$. To be more precise, define $g(B)$ inductively as follows:

$$
\begin{aligned}
& g\left(\bigcirc^{n} B\right)=\bigcirc^{n} B, \text { if } B \in P V, \\
& g\left(\bigcirc^{n} \neg B\right)=\neg g\left(\bigcirc^{n} B\right), \\
& g\left(\bigcirc^{n}(B \vee C)\right)=g\left(\bigcirc^{n} B\right) \vee g\left(\bigcirc^{n} C\right), \text { and } \\
& g\left(\bigcirc^{n} \square B\right)=\square g\left(\bigcirc^{n} B\right) .
\end{aligned}
$$

Definition 28. A near-atom is a formula of the form $\bigcirc^{n} p$ where $p \in P V$.

Definition 29. A formula is simple iff it is built up from near-atoms using the Boolean connectives and $\square$. Simple formulas are the formulas in the range of $g$.

Convention 30. We will take S4 to be formulated by its standard axioms and rules, for a language whose formulas are just the simple formulas, treating the near-atoms as indivisible atomic formulas. We also slightly restate the definition of topological model, 
Definition 1: A topological model now becomes an ordered pair, $M=\langle X, V\rangle$, where $X$ is a topological space and $V$ assigns a subset of $X$ to each near-atom $\bigcirc^{n} p$ rather than to each propositional variable $p$. Mimicking Definition 1 , for each simple formula $B$, we define $M(B)$, the subset assigned by $M$ to $B$ as follows:

$$
\begin{aligned}
& M\left(\bigcirc^{n} p\right)=V\left(\bigcirc^{n} p\right), \\
& M(A \vee B)=M(A) \cup M(B), \\
& M(\neg B)=X-M(B), \text { and } \\
& M(\square B)=\operatorname{Int}(M(B)) .
\end{aligned}
$$

As in Definition 1, we define standard validity relations:

$$
\begin{aligned}
& M \models B \text { iff } M(B)=X . \\
& X \models B \text { iff } M \models B \text { for every model } M=\langle X, V\rangle .
\end{aligned}
$$

$B$ is valid $(\models B)$ iff $X \models B$ for every topological space $X$.

The McKinsey-Tarski-Kripke Theorem 10 still holds: Suppose that $X$ is a dense-initself metric space and $A$ is a simple formula. Then the following are equivalent: (i) $A \in S 4$; (ii) $\models A$; (iii) $X \models A$; (iv) $\mathbb{R} \models A$; (v) $Y \models A$ for every finite topological space $Y$; and (vi) $Y \models A$ for every Alexandrov space $Y$.

Lemma 31. $B \in \mathrm{S} 4 \bigcirc$ iff $g(B) \in \mathrm{S} 4$ iff $g(B) \in \mathrm{S} 4 \bigcirc$.

Proof. By a standard induction on the proof of $B$ in $\mathrm{S} 4 \bigcirc$, we can show that if $B \in \mathrm{S} 4 \bigcirc$ then $g(B) \in \mathrm{S} 4$. It is obvious that if $g(B) \in \mathrm{S} 4$ then $g(B) \in \mathrm{S} 4 \bigcirc$. Finally, if $g(B) \in \mathrm{S} 4 \bigcirc$ then $B \in \mathrm{S} 4 \bigcirc$, since $(B \equiv g(B)) \in \mathrm{S} 4 \bigcirc$.

Lemma 32. For every formula $B, g(B) \in \mathrm{S} 4$ iff $(0,1) \models g(B)$ where $(0,1)$ is the open unit interval.

Proof. This follows from the McKinsey-Tarski-Kripke Theorem 10 and Lemma 31.

Theorem 33. If $\mathbb{R}, \mathcal{H} \models A$ then $A \in \mathrm{S} 4 \bigcirc$.

Proof. Suppose that $A \notin \mathrm{S} 4 \bigcirc$. Then, by Lemmas 31 and 32, for some topological model $M=\langle(0,1), V\rangle$, we have $M \not \forall g(A)$. Let $M^{\prime}$ be the dynamic topological model $\left\langle\mathbb{R}, f, V^{\prime}\right\rangle$, where $f x=x+1$ and $V^{\prime}(p)=\{x \in \mathbb{R}$ : for some natural number $m$, $\left.x-m \in V\left(\bigcirc^{m} p\right)\right\} . f$ is a homeomorphism. We will be done if we can show that $M^{\prime} \not \models A$. For this, it suffices to show that $M^{\prime} \not \forall g(A)$, because of Lemma 31 and because of soundness. And for this it suffices to show that for every simple formula $B$, we have $M(B)=(0,1) \cap M^{\prime}(B)$. We show this by induction on the construction of $B$.

Base case: $B$ is a near-atom, say $\bigcirc^{n} p$. Note the following:

$$
\begin{aligned}
& x \in(0,1) \cap M^{\prime}(B) \\
& \Rightarrow x \in(0,1) \text { and } x \in M^{\prime}\left(\bigcirc^{n} p\right) \\
& \Rightarrow x \in(0,1) \text { and } x+n \in M^{\prime}(p) \\
& \left.\Rightarrow x \in(0,1) \text { and } x+n \in V^{\prime}(p)\right]
\end{aligned}
$$




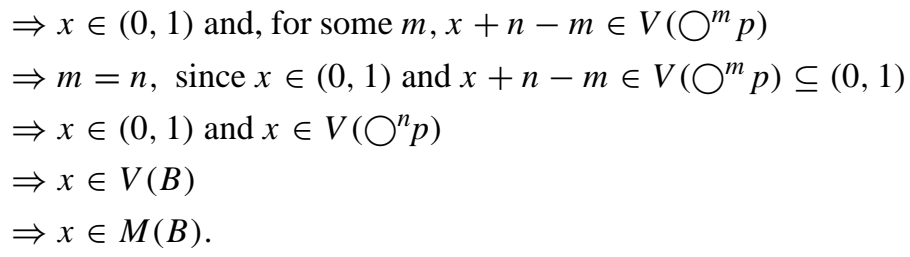

Conversely,

$$
\begin{aligned}
& x \in M(B) \\
& \Rightarrow x \in V(B) \\
& \Rightarrow x \in(0,1) \text { and } x \in V\left(\bigcirc^{n} p\right) \\
& \Rightarrow x \in(0,1) \text { and, for some } m, x+n-m \in V\left(\bigcirc^{m} p\right) \\
& \Rightarrow x \in(0,1) \text { and } x+n \in V^{\prime}(p) \\
& \Rightarrow x \in(0,1) \text { and } x+n \in M^{\prime}(p) \\
& \Rightarrow x \in(0,1) \text { and } x \in M^{\prime}\left(\bigcirc^{n} p\right) \\
& \Rightarrow x \in(0,1) \cap M^{\prime}(B) .
\end{aligned}
$$

Inductive step $B=C \vee D . M(C \vee D)=M(C) \cup M(D)=\left((0,1) \cap M^{\prime}(C)\right) \cup((0,1) \cap$ $\left.M^{\prime}(D)\right)=(0,1) \cap M^{\prime}(C \vee D)$.

Inductive step $B=\neg C . M(\neg C)=(0,1)-M(C)=(0,1)-\left((0,1) \cap M^{\prime}(C)\right)=$ $(0,1)-\left(\mathbb{R} \cap M^{\prime}(C)\right)=(0,1) \cap M^{\prime}(\neg C)$.

Inductive step $B=\square C \cdot M(\square C)=\operatorname{Int}(M(C))=\operatorname{Int}\left((0,1) \cap M^{\prime}(C)\right)=\operatorname{Int}((0,1)) \cap$ $\operatorname{Int}\left(M^{\prime}(C)\right)=(0,1) \cap M^{\prime}(\square C)$.

Theorem 34. If $\mathcal{A}, \mathcal{H} \models A$ then $A \in \mathrm{S} 4 \bigcirc$.

Proof. Suppose that $A \notin \mathrm{S} 4 \bigcirc$. Then $g(A) \notin \mathrm{S} 4$. So there is a Kripke model $M=$ $\langle W, R, V\rangle$ (where $\langle W, R\rangle$ is a Kripke frame) such that $M \not \models g(A)$. Now define a dynamic topological model $M^{\prime}=\left\langle X, f, V^{\prime}\right\rangle$ as follows:

$X=\{\langle w, n\rangle: w \in W$ and $n$ is an integer $\}$,

$\langle w, n\rangle R^{\prime}\left\langle w^{\prime}, m\right\rangle$ iff $w R w^{\prime}$ and $n=m$,

$Y \subseteq X$ is open iff $Y$ is closed under the relation $R^{\prime}$,

$f\langle w, n\rangle=\langle w, n+1\rangle$, and

$\langle w, n\rangle \in V^{\prime}(p)$ iff $w \in V\left(\bigcirc^{n} p\right)$.

$X$ is a topological space, if we take the topology of open sets as defined directly above. In fact, $X$ is an Alexandrov space (see Definition 4). $f$ is both continuous and open since $\langle w, n\rangle R^{\prime}\left\langle w^{\prime}, m\right\rangle$ iff $f\langle w, n\rangle R^{\prime} f\left\langle w^{\prime}, m\right\rangle$. And $f$ is clearly one-one and onto. So $M^{\prime}=\left\langle X, f, V^{\prime}\right\rangle$ is a dynamic Alexandrov model, with $f$ a homeomorphism. We will be done if we can show that $M^{\prime} \not \models A$. For this, it suffices to show that $M^{\prime} \not \models g(A)$, because of Lemma 31 and because of soundness. And for this it suffices to show that for every 
simple formula $B$ and every $w \in W$ we have $w \in M(B)$ iff $\langle w, 0\rangle \in M^{\prime}(B)$. We show this by induction on the construction of $B$.

Base case: $B$ is a near-atom, say $\bigcirc^{n} p$. Then $\langle w, 0\rangle \in M^{\prime}(B)$ iff $\langle w, 0\rangle \in M^{\prime}\left(\bigcirc^{n} p\right)$ iff $f^{n}\langle w, 0\rangle \in M^{\prime}(p)$ iff $\langle w, n\rangle \in M^{\prime}(p)$ iff $\langle w, n\rangle \in V^{\prime}(p)$ iff $w \in V\left(\bigcirc^{n} p\right)$ iff $w \in M\left(\bigcirc^{n} p\right)$ iff $w \in M(B)$.

Inductive step $B=C \vee D .\langle w, 0\rangle \in M^{\prime}(C \vee D)$ iff $\langle w, 0\rangle \in M^{\prime}(C)$ or $\langle w, 0\rangle \in M^{\prime}(D)$ iff $w \in M(C)$ or $w \in M(D)$ iff $w \in M(C \vee D)$.

Inductive step $B=\neg C .\langle w, 0\rangle \in M^{\prime}(\neg C)$ iff $\langle w, 0\rangle \notin M^{\prime}(C)$ iff $w \notin M(C)$ iff $w \in M(\neg C)$.

Inductive step $B=\square C .\langle w, 0\rangle \in M^{\prime}(\square C)$ iff $\left(\forall w^{\prime}\right)(\forall n)$ (if $\langle w, 0\rangle R^{\prime}\left\langle w^{\prime}, n\right\rangle$ then $\left\langle w^{\prime}, n\right\rangle \in$ $\left.M^{\prime}(C)\right)$ iff $\left(\forall w^{\prime}\right)$ (if $\left(w R w^{\prime}\right.$ then $\left.\left\langle w^{\prime}, 0\right\rangle \in M^{\prime}(C)\right)$ iff $\left(\forall w^{\prime}\right)\left(\right.$ if $w R w^{\prime}$ then $\left.w^{\prime} \in M(C)\right)$ iff $w \in M(\square C)$.

Given Theorem 25, one might conjecture that

$$
\begin{aligned}
& \operatorname{DTL}_{\mathcal{H}}=\operatorname{DTL}_{\mathbb{R}, \mathcal{H}}=\operatorname{DTL}_{[0,1], \mathcal{H}}=\operatorname{DTL}_{\mathcal{A}, \mathcal{H}}= \\
& \operatorname{DTL}_{\mathcal{O}}=\operatorname{DTL}_{\mathbb{R}, \mathcal{O}}=\operatorname{DTL}_{[0,1], \mathcal{O}}=\operatorname{DTL}_{\mathcal{A}, \mathcal{O}}
\end{aligned}
$$

But [24] defines a formula $A \in \mathrm{DTL}_{\mathbb{R}, \mathcal{H}}-\mathrm{DTL}_{\mathcal{H}}$. Moreover, the example at the beginning of Section 3 shows that

$$
(* \square p \supset \square * p) \in \mathrm{DTL}_{\mathcal{A}, \mathcal{H}}-\mathrm{DTL}_{\mathcal{H}} .
$$

Nonetheless, we propose the following:

Conjecture 35. (i) $\mathrm{DTL}_{\mathcal{H}}=\mathrm{DTL}_{\mathcal{O}}$. (ii) $\mathrm{DTL}_{\mathbb{R}, \mathcal{H}}=\mathrm{DTL}_{[0,1], \mathcal{H}}=\mathrm{DTL}_{\mathbb{R}, \mathcal{O}}=$ $\operatorname{DTL}_{[0,1], \mathcal{O}}$. (iii) $\operatorname{DTL}_{\mathcal{A}, \mathcal{H}}=\operatorname{DTL}_{\mathcal{A}, \mathcal{O}}$.

\section{Recurrence and the DTL of measure-preserving continuous functions on the closed unit interval}

A central motivation for this study is the phenomenon of recurrence in measure theory and topological dynamics, and the possibility of expressing this phenomenon in the framework of propositional logic. In fact, we can express recurrence in our trimodal language.

Suppose that $f$ is a function on a set $X$. Say that a point $x \in S$ is recurrent (for $S$ ) if $f^{n}(x) \in S$ for some $n>1$. Let $\mu$ be the Lebesgue measure defined on (some) subsets of the closed unit interval, $[0,1]$. If $\mu(S)$ exists for $S \subseteq[0,1]$, we say that $S$ is measurable. We say that a function $f$ on $[0,1]$ is measure-preserving iff $\mu\left(f^{-1}(S)\right)=\mu(S)$ for every measurable $S \subseteq[0,1]$. Consider the following (non-essential) extension of the Poincaré recurrence theorem on $[0,1]$ (see [28]):

Theorem 36. If $f$ is a measure-preserving continuous function on $[0,1]$ then the set of recurrent points of a non-empty open set $S \subseteq[0,1]$ is dense in $S$. 
In order to express recurrence in our trimodal language, define the possibility connective $\diamond$ as $\neg \square \neg$, and the possibility connective \# as $\neg * \neg$. These represent topological closure and "some time in the future", respectively. Let rec be the formula,

$$
(\square p \supset \diamond \bigcirc \# \square p) \text {. }
$$

Let $\langle X, f\rangle$ be any dynamic topological system. Note that $\langle X, f\rangle \models$ rec iff

$$
\forall \text { open } O \subseteq X: O \subseteq C l\left\{x: \text { there is an } n \geq 1 \text { such that } f^{n} x \in O\right\} .
$$

By Theorem 36, $(*)$ is true when $X=[0,1]$ and $f$ is any measure-preserving continuous function on $[0,1]$. Thus, by Theorem $36,\langle[0,1], f\rangle \vDash$ rec when $f$ is any measurepreserving continuous function on $[0,1]$. So, in some sense, rec expresses the phenomenon of recurrence.

Thus the class $\mathcal{M}$ of measure-preserving functions on the $[0,1]$ is of interest. As we have just shown,

$(\square A \supset \diamond \bigcirc \# \square A) \in \mathrm{DTL}_{\mathcal{M}}$.

Conjecture 37. $\mathrm{DTL}_{\mathcal{M}}=\mathrm{DTL}_{[0,1]}+(\square A \supset \diamond \bigcirc \# \square A)$.

\section{Purely topological and purely temporal fragments of DTLs}

In work on DTL, we foresee that most of the action will be in the interaction between the topological modality $(\square)$ and the temporal modalities ( $\bigcirc$ and $*$ ). As it turns out, temporal differences often do not affect purely topological issues (see Theorem 38). Furthermore, the purely topological fragments and the purely temporal fragments of DTLs will often coincide with previously studied logics (see Theorems 39 and 43).

Theorem 38. Suppose that $\mathcal{T}$ is a class of topological spaces and $\mathcal{F}$ is a class of continuous functions. Also suppose that for every $X \in \mathcal{T}$, there is an $f \in \mathcal{F}$ with $\operatorname{dom}(f)=X$. Then $\mathrm{DTL}_{\mathcal{T}, \mathcal{F}}^{\square}=\mathrm{DTL}_{\mathcal{\mathcal { T }}}^{\square}$. Thus temporal differences do not affect purely topological issues.

Theorem 39. Suppose that $\mathcal{T}$ is a class of topological spaces and that either

(i) every topological space is in $\mathcal{T}$,

(ii) $\mathbb{R} \in \mathcal{T}$,

(iii) some dense-in-itself metric space is in $\mathcal{T}$,

(iv) every finite topological space is in $\mathcal{T}$, or

(v) every Alexandrov space is in $\mathcal{T}$.

Then $\mathrm{DTL}_{\mathcal{\mathcal { T }}}^{\square}=\mathrm{S} 4$.

Proof. This follows from the McKinsey-Tarski-Kripke Theorem 10.

Corollary 40. $\mathrm{DTL}_{0}^{\square}=\operatorname{DTL}_{\mathcal{H}}^{\square}=\operatorname{DTL}_{\mathcal{M}}^{\square}=\operatorname{DTL}_{\mathcal{A}}^{\square}=\operatorname{DTL}_{\mathbb{R}}^{\square}=\operatorname{DTL}_{[0,1]}^{\square}=\operatorname{DTL}_{\mathbb{R}, \mathcal{H}}^{\square}=$ $\mathrm{DTL}_{\mathcal{A}, \mathcal{H}}^{\square}=\mathrm{DTL}_{\text {fin }}^{\square}=\mathrm{S} 4$, where fin is the class of finite topological spaces. (Such examples are easily multiplied.) 
Definition 41. Suppose that $f$ is a continuous function and that $X=\operatorname{dom}(f)$. For $m, n \in \omega, f$ has the $m-n$-property iff there is some $x \in X$ such that $x, f x, \ldots, f^{m+n} x$ are all distinct and $f^{m+n+1} x=f^{m} x . f$ has the $\omega$-property iff there is some $x \in X$ such that $x, f x, f^{2} x, \ldots$ are all distinct. Suppose that $\mathcal{F}$ is a class of continuous functions. $\mathcal{F}$ is rich iff either (i) $\mathcal{F}$ contains some function with the $\omega$-property or (ii) for each $m, n \in \omega$, $\mathcal{F}$ contains some function with the $m-n$-property.

Remark 42. The following classes of functions are rich:

(i) the class $\mathcal{H}$ of homeomorphisms;

(ii) the class $\mathcal{O}$ of open continuous functions (a function is open iff the image of every open set is open);

(iii) the class $\mathcal{M}$ of measure-preserving continuous functions on [0, 1]; and

(iv) the class of functions on finite topological spaces with the discrete topology.

For (i) and (ii) it suffices to find a homeomorphism on $\mathbb{R}$ with the $\omega$-property, for example $f x=x+1$. For (iii), the following function is continuous, measure-preserving, and has the $\omega$-property: $f(x)=1-2 x$ for $x \in\left[0, \frac{1}{2}\right]$ and $f(x)=2 x-1$ for $x \in\left[\frac{1}{2}, 1\right]$. To see that $f$ is measure-preserving consider any $S \subseteq[0,1]$. Note that $\mu\left(f^{-1}(S) \cap\left[0, \frac{1}{2}\right]\right)=$ $\mu\left(f^{-1}(S) \cap\left[\frac{1}{2}, 1\right]\right)=\frac{1}{2} \mu(S)$, so $\mu\left(f^{-1}(S)\right)=\mu(S)$. To see that $f$ has the $\omega$-property, let $x=\sqrt{2}-1$. Note that $f^{n}(x)$ is of the form $z \pm 2^{n} \sqrt{2}$, where $z$ is an integer, so $x, f x, f^{2} x, \ldots$ are all distinct. For (iv), we fix $m$ and $n$ and define a function with the $m-$ $n$-property in the given class. Let $X$ be the set $\{0,1,2, \ldots, m+n\}$ and let $f x=x+1$ if $x<m+n$ and let $f(m+n)=m$.

Theorem 43. Suppose that $\mathcal{F}$ is a rich class of continuous functions. Then $\mathrm{DTL}_{\mathcal{F}}^{\bigcirc *}=\mathrm{W} 0$.

Proof. Recall the axiomatization of W0 given in the introduction. To show that W0 $\subseteq$ DTL $\mathcal{F}_{\mathcal{F}}^{\circ}$, it suffices to show that this axiomatization is sound for $\mathrm{DTL}_{\mathcal{F}}^{\bigcirc *}$. To show that DTL $\mathcal{F}_{\mathcal{F}} *$ W0, we consider two cases.

Case 1. $\mathcal{F}$ contains a function with the $\omega$-property. Suppose that $A \notin \mathrm{W} 0$ where $A$ is in the language $L^{\bigcirc *}$. Then there is some infinite purely temporal model falsifying $A$. To be more precise, let an infinite purely temporal model be a function $V: P V \times \omega \rightarrow\{0,1\}$, where $P V$ is the set of propositional variables; where the natural numbers represent discrete moments in time; and where 0 and 1 represent falsity and truth. Given an infinite purely temporal model $V$, we define $n=B$, for each $n \in \omega$ and each formula $B$ in the language $L^{\circ *}$ as follows: $n \models p$ iff $V(p, n)=1 ; n \models \neg B$ iff $n \not \models B ; n \models(B \vee C)$ iff $n \models B$ or $n \models C ; n \models \bigcirc B$ iff $n+1 \models B$; and $n \models * B$ iff $m \models B$ for every $m \geq n$. The completeness theorem for W0 tells us that since $A \notin \mathrm{W} 0$, there is some infinite purely temporal model $V$ such that $0 \not \forall A$. Choose such a $V$.

Since $\mathcal{F}$ contains a function with the $\omega$-property, we can choose a topological space $X$ and a function $f \in \mathcal{F}$ and an $x \in X$, such that the points $x, f x, f f x, f f f x, \ldots$ are all distinct. Choose a function $V^{\prime}: P V \rightarrow X$ such that $f^{k} x \in V^{\prime}(p)$ iff $V(p, k)=1$, for every $k \in \omega$. And define $M=\left\langle X, T, V^{\prime}\right\rangle$. By a standard induction on formulas, it can be shown that $f^{k} x \in M(B)$ iff $k \models B$ for all formulas $B$ in the language $L^{\bigcirc *}$ and all $k \in \omega$. Thus $x \not \models A$ since $0 \not \models A$. So $A \notin \mathrm{DTL}_{\mathcal{F}}^{\bigcirc *}$, as desired. 
Case 2. $\mathcal{F}$ contains a function with the $m-n$-property for every $m, n \in \omega$. Suppose that $A \notin$ W0 where $A$ is in the language $L^{\bigcirc *}$. Let a finite purely temporal model be an ordered triple $M=\langle Y, g, V\rangle$ where $Y$ is a finite set; $g$ is a function on $Y$; and $V: P V \times Y \rightarrow\{0,1\}$. Given a finite purely temporal model $M=\langle Y, g, V\rangle$, we define $y \models B$, for each $y \in Y$ and each formula $B$ in the language $L^{\bigcirc^{*}}$ as follows: $y \models p$ iff $V(p, y)=1 ; y \models \neg B$ iff $y \not \models B ; y \models(B \vee C)$ iff $y \models B$ or $y \models C ; y \models \bigcirc B$ iff $g(y) \models B$; and $y \models * B$ iff $g^{n}(y) \models B$ for every $n \geq 0$.

Reference [21] proves that W0 satisfies the finite frame property. So since $A \notin \mathrm{W} 0$, there is some finite purely temporal model $M=\langle Y, g, V\rangle$ and some $y \in Y$ such that $y \not \models A$. Since $Y$ is finite, we have $g^{m+n+1}(y)=g^{m}(y)$, for some $m, n \in \omega$ with the $g^{i}(y)$ distinct for $i<m+n$. Choose such an $m$ and $n$.

Choose a function $f \in \mathcal{F}$ with the $m-n$-property and let $X$ be the topological space on which $f$ acts. Choose an $x \in X$ such that $x, f x, \ldots, f^{m+n} x$ are all distinct, and such that $f^{m+n+1} x=f^{m} x$. Define $V^{\prime}: P V \rightarrow \mathcal{P}(X)$ as follows:

$$
V^{\prime}(p)=\left\{f^{k} x: V\left(p, g^{k} y\right)=1\right\} .
$$

And let $M^{\prime}=\left\langle X, f, V^{\prime}\right\rangle$. Claim: $f^{k} x \in M^{\prime}(B)$ iff $g^{k} y \models B$ for all $k \in \omega$ and formulas $B$ in the language $L^{O *}$. We prove this by induction on formulas.

Base case: For propositional variables $p: f^{k} x \in M^{\prime}(p)$ iff $f^{k} x \in V^{\prime}(p)$ iff $V\left(p, g^{k} y\right)=1$ iff $g^{k} y \models p$.

Inductive step $\neg, \vee$ : standard.

Inductive step $B=\bigcirc C: f^{k} x \in M^{\prime}(\bigcirc C)$ iff $f^{k+1} x \in M^{\prime}(C)$ iff $g^{k+1} y \models C$ (by IH) iff $g^{k} x \models \bigcirc C$.

Inductive step $B=* C: f^{k} x \in M^{\prime}(* C)$ iff $(\forall n \geq k)\left(f^{n} x \in M^{\prime}(C)\right)$ iff $(\forall n \geq k)\left(g^{n} y \models\right.$ C) (by IH) iff $g^{k} y=* C$.

Thus $x \notin M^{\prime}(A)$ since $y \not \models A$. So $A \notin \mathrm{DTL}_{\mathcal{F}}^{\bigcirc *}$, as desired.

Corollary 44. $\mathrm{DTL}_{0}^{\bigcirc *}=\mathrm{DTL}_{\mathcal{H}}^{\bigcirc *}=\mathrm{DTL}_{\mathcal{M}}^{\bigcirc *}=\mathrm{DTL}_{\mathcal{A}}^{\bigcirc *}=\mathrm{DTL}_{\mathbb{R}}^{\bigcirc *}=\mathrm{DTL}_{[0,1]}^{\bigcirc *}=$ $\mathrm{DTL}_{\mathbb{R}, \mathcal{H}}^{\bigcirc *}=\mathrm{DTL}_{\mathcal{A}, \mathcal{H}}^{\bigcirc *}=\mathrm{DTL}_{\text {fin }}^{\bigcirc *}=\mathrm{W} 0$, where fin is the class of finite topological spaces. (Such examples are easily multiplied.)

\section{A temporal-over-topological fragment}

In this section we axiomatize $\mathrm{DTL}_{0}^{\bigcirc * / \square}$, the temporal-over-topological fragment of $\mathrm{DTL}_{0}$. Recall that

$$
\mathrm{DTL}_{0}^{\bigcirc * / \square}={ }_{\mathrm{df}}\left\{A \in \mathrm{DTL}_{0}: A \text { contains no } \bigcirc \text { 's or } * \text { 's in the scope of } \square \text { 's }\right\} .
$$

For the remainder of this section, we build the scope constraint into the definition of wellformed formulas.

Let the logic W0/S4 be the logic given by the following axiomatic system: 
0. Classical tautologies

1. S4 axioms for $\square$, for formulas $A$ and $B$ in the language $L^{\square}$ :

$$
\begin{aligned}
& 1.1 \square(A \supset B) \supset(\square A \supset \square B) \\
& 1.2 \square A \supset A \\
& 1.3 \square A \supset \square \square A
\end{aligned}
$$

2. W0 axioms for $\bigcirc$ and $*$ :

$$
\begin{aligned}
& 2.1 *(A \supset B) \supset(* A \supset * B) \\
& 2.2 * A \supset A \\
& 2.3 * A \supset * * A \\
& 2.4(\bigcirc(A \vee B) \equiv(\bigcirc A \vee \bigcirc B)) \\
& 2.5(\bigcirc \neg A \equiv \neg \bigcirc A) \\
& 2.6(\bigcirc * A \equiv * \bigcirc A) \\
& 2.7(* A \supset \bigcirc A)
\end{aligned}
$$

2.8 The induction axiom $(A \& *(A \supset \bigcirc A) \supset * A)$

3. The rule of modus ponens.

4. The rule of necessitation for each modality: From $A$ infer $\bigcirc A$. From $A$ infer $* A$. From $A$ infer $\square A$, if $A$ has no occurrences of $\bigcirc$ or $*$.

We will say that $A$ is a theorem iff $A \in \mathrm{W} 0 / \mathrm{S} 4$ and that $A$ is consistent iff $\neg A \notin \mathrm{W} 0 / \mathrm{S} 4$. A W0/S4-theory is a set of formulas containing all the theorems of W0/S4 and closed under modus ponens (and hence, under adjunction). $A$ is an S4-theorem iff $A$ has no occurrence of $\bigcirc$ or $*$ and $A \in \mathrm{S} 4$. An S4-theory is a set of formulas in the language $L^{\square}$, containing all the theorems of $\mathrm{S} 4$ and closed under modus ponens (and hence, under adjunction). A W0/S4-theory $T$ is complete iff for every formula $A$ either $A \in T$ or $\neg A \in T$. A W0/S4theory $T$ is consistent iff some formula is not in $T$. A set $S$ of formulas is consistent iff some theory $T \supseteq S$ is consistent. A W0/S4-theory $T$ is $\omega$-closed iff, for any formula $A$, we have the following: if $\bigcirc^{n} A \in T$ for every $n \in \omega$ then $* A \in T$.

The main result of this section is

Theorem 45. W0/S4 $=\mathrm{DTL}_{0}^{\bigcirc * / \square}$.

Proving soundness (W0/S4 $\subseteq \mathrm{DTL}_{0}^{\bigcirc * / \square}$ ) is routine and left to the reader. Proving completeness ( $\mathrm{DTL}_{0}^{\bigcirc * / \square} \subseteq \mathrm{W} 0 / \mathrm{S} 4$ ) is a bit tricky. We proceed as follows: we introduce some notation and terminology; we state six useful lemmas, whose proofs we defer; we state and prove completeness; and we provide the deferred proofs of the lemmas.

First some notation: \#A is shorthand for $\neg * \neg A$. Secondly, some terminology. A necessitive is a formula of the form $\square$. A quasi-necessitive is a formula of the form 
$\bigcirc^{n_{1}} \square A_{1} \& \ldots \& \bigcirc^{n_{m}} \square A_{m}, n_{i} \geq 0$. The degree of a quasi-necessitive $\bigcirc^{n_{1}} \square A_{1}$ \& $\ldots \& \bigcirc^{n_{m}} \square A_{m}$ is $\min \left(n_{1}, \ldots, n_{m}\right)$. Given a quasi-necessitive $A=\bigcirc^{n_{1}} \square A_{1} \& \ldots$ \& $\bigcirc^{n_{m}} \square A_{m}$, we define the quasi-necessitive $\uparrow A={ }_{\mathrm{df}} \bigcirc^{n_{1}+1} \square A_{1} \& \ldots$ \& \& $\bigcirc^{n_{m}+1} \square A_{m}$. Given a quasi-necessitive $A=\bigcirc^{n_{1}} \square A_{1} \& \ldots \& \bigcirc^{n_{m}} \square A_{m}$ of degree $\geq 1$, we define the quasi-necessitive $\downarrow A={ }_{\text {df }} \bigcirc^{n_{1}-1} \square A_{1} \& \ldots \& \bigcirc^{n_{m}-1} \square A_{m}$.

Our six useful lemmas are Lemmas 46 to 51 as follows:

Lemma 46. Suppose that $(A \vee \bigcirc B) \in \mathrm{W} 0 / \mathrm{S} 4$ and that $A$ contains no occurrences of $\bigcirc$ or $*$. Then $A \in \mathrm{S} 4$ or $B \in \mathrm{W} 0 / \mathrm{S} 4$.

Lemma 47. Suppose that $(A \supset B) \in \mathrm{W} 0 / \mathrm{S} 4$ and that $B$ contains no occurrences of $\bigcirc$ or * and that $A$ is a quasi-necessitive. Then $(A \supset \square B) \in \mathrm{W} 0 / \mathrm{S} 4$.

Lemma 48. Suppose that $\bigcirc A \in \mathrm{W} 0 / \mathrm{S} 4$. Then $A \in \mathrm{W} 0 / \mathrm{S} 4$.

Lemma 49. If $(A \& \# B)$ is consistent, then $\left(A \& \bigcirc^{n} B\right)$ is consistent for some $n \in \omega$.

Lemma 50. If $A$ is consistent then $A \in T$ for some consistent complete $\omega$-closed W0/S4theory $T$.

Lemma 51. $(A \supset B) \in \mathrm{W} 0 / \mathrm{S} 4$ iff for every consistent complete $\omega$-closed $\mathrm{W} 0 / \mathrm{S} 4$-theory $T$, if $A \in T$ then $B \in T$.

Using these lemmas, we can prove completeness.

Theorem 52. $\mathrm{DTL}_{0}^{\bigcirc * / \square} \subseteq \mathrm{W} 0 / \mathrm{S} 4$.

Proof. It will suffice to define a canonical dynamic topological model $M=\langle X, f, V\rangle$ validating all and only the theorems of W0/S4. To define $M$,

(i) let $X$ be the set of complete consistent $\omega$-closed theories;

(ii) given a quasi-necessitive $A$, let $\mathbf{B}_{A}=\{u \in X: A \in u\}$;

(iii) impose the topology on $X$ given by the basis sets $\mathbf{B}_{A}$, where $A$ is a quasi-necessitive; the $\mathbf{B}_{A}$ form a basis since they are closed under intersection: $\mathbf{B}_{A} \cap \mathbf{B}_{C}=\mathbf{B}_{(A \& C)}$;

(iv) given $u \in X$, let $f u=\{A: \bigcirc A \in u\}$; and

(v) let $V(p)=\{u \in X: p \in u\}$.

First we check that $M$ is indeed a dynamic topological model. We will check two things: (1) $f u \in X$ for each $u \in X$; and (2) $f$ is continuous. For (1), suppose that $u$ is a complete consistent $\omega$-closed W0/S4-theory. To see that $f u$ is a theory, first note that $f u$ contains every theorem: if $A$ is a theorem then so is $\bigcirc A$; so $\bigcirc A \in u$; so $A \in f u$. And furthermore note that $f u$ is closed under modus ponens:

$$
\begin{aligned}
& (A \supset B) \in f u \text { and } A \in f u \\
& \Rightarrow \bigcirc(A \supset B) \in u \text { and } \bigcirc A \in u \\
& \Rightarrow(\bigcirc A \supset \bigcirc B) \in u \text { and } \bigcirc A \in u \\
& \Rightarrow \bigcirc B \in u \\
& \Rightarrow B \in f u .
\end{aligned}
$$


To see that $f u$ is complete, note that for any formula $A$ we have $\bigcirc A \in u$ or $\neg \bigcirc A \in u$; so $\bigcirc A \in u$ or $\bigcirc \neg A \in u$; so $A \in f u$ or $\neg A \in f u$. To see that $f u$ is consistent, suppose not. Then for some formula $A \in f u$, we have $\neg A \in$ W0/S4. But then $\bigcirc A \in u$ and $\bigcirc \neg A \in \mathrm{W} 0 / \mathrm{S} 4$, so $\bigcirc A \in u$ and $\neg \bigcirc A \in \mathrm{W} 0 / \mathrm{S} 4$. This would make $u$ itself inconsistent. To see that $f u$ is $\omega$-closed, fix a formula $A$ and suppose that $\bigcirc^{n} A \in f u$ for every $n$. Then $\bigcirc^{n+1} A \in u$ for every $n$. So $* \bigcirc A \in u$ by the $\omega$-closure of $u$. So $\bigcirc * A \in u$. So $* A \in f u$. So $f u$ is an $\omega$-closed complete consistent theory. Thus (1) is proved: $f u \in X$ for each $u \in X$.

For (2), i.e. the continuity of $f$, it suffices to note that $f^{-1}\left(\mathbf{B}_{A}\right)=\mathbf{B}_{\uparrow A}$. So $M$ is indeed a dynamic topological model.

We now prove that, for each formula $A$ and each $x \in X$, we have :

$$
x \in M(A) \text { iff } A \in x .
$$

We proceed by induction.

Base case: $A \in P V$. Note: $x \in M(A)$ iff $x \in V(A)$ iff $A \in x$, by the definition of $V$.

Inductive step $A=\neg B$. Note: $x \in M(A)$ iff $x \notin M(B)$ iff $B \notin x$ (by IH) iff $A \in x$, by the completeness of the theory $x$.

Inductive step $A=B \vee C$. Note: $x \in M(A)$ iff $x \in M(B)$ or $x \in M(C)$ iff $B \in x$ or $C \in x$ (by IH) iff $A \in x$. The $(\Rightarrow)$ direction of this last 'iff' follows from the fact that $x$ is a W0/S4-theory. The $(\Leftarrow)$ direction follows from the completeness of the theory $x$.

Inductive step $A=\bigcirc C$. Note: $x \in M(A)$ iff $f x \in M(C)$ iff $C \in f x$ (by IH) iff $A \in x$, by the definition of $f$.

Inductive step $A=* C$. Note: $x \in M(* C)$ iff $(\forall n \in \omega)\left(f^{n} x \in M(C)\right)$ iff $(\forall n \in \omega)(C \in$ $\left.f^{n} x\right)$ (by IH) iff $(\forall n \in \omega)\left(\bigcirc^{n} C \in x\right)$ (by the definition of $\left.f\right)$ iff $* C \in x$ (by the $\omega$-closure of $x$ ).

Inductive step $A=\square C$. We consider both directions of the biconditional separately. $(\Rightarrow)$ Suppose that $x \in M(\square C)=\operatorname{Int}(M(C))$. Then for some basis set $\mathbf{B}_{D}$, where $D$ is a quasinecessitive, we have $x \in \mathbf{B}_{D} \subseteq M(C)$. So $D \in x$. Moreover, for every $y \in X$, if $D \in y$ then $y \in M(C)$, in which case $C \in y$, by IH. So $(D \supset C) \in \mathrm{W} 0 / \mathrm{S} 4$, by Lemma 51 . So $(D \supset \square C) \in \mathrm{W} 0 / \mathrm{S} 4$, by Lemma 47. So $\square C \in x$, as desired. $(\Leftarrow)$ Suppose that $\square C \in x$. It suffices to show that $x \in \mathbf{B}_{\square C} \subseteq M(C)$ in order to show that $x \in \operatorname{Int}(M(C))=M(\square C)$. $x \in \mathbf{B}_{\square C}$ is given by the definition of $\mathbf{B}_{\square C}$. For $\mathbf{B}_{\square C} \subseteq M(C)$, suppose that $y \in \mathbf{B}_{\square C}$. Then $\square C \in y$. So $C \in y$ as desired.

Having proved ( $*$ ), the last step in the Completeness proof is to note that, if $A \notin \mathrm{W} 0 / \mathrm{S} 4$, then, by Lemma 50, for some $y \in X$ we have $A \notin y$. So $y \notin M(A)$. So $M \not \models A$. So $A \notin$ $\mathrm{DTL}^{\bigcirc} / \square$.

Now it remains to prove Lemmas 46 to 51 .

Proof of Lemma 46. The proof is semantic. Let a birelational model be a quartuple $M=\langle W, S, R, V\rangle$ where $W$ is a non-empty set (of possible worlds); $S$ and $R$ are binary relations on $W$; and $V$ assigns to each possible world a complete consistent S4-theory in 
the language $L^{\square}$. Given a birelational model $M=\langle W, S, R, V\rangle$, we define the validation relation $\models$ between worlds and formulas as follows:

$$
\begin{aligned}
& w \models C \text { iff } C \in V(w), \text { if } C \text { is in the language } L^{\square} ; \\
& w \models \neg C \text { iff } w \not \models C ; \\
& w \models(C \vee D) \text { iff } w \models C \text { or } w \models D ; \\
& w \models \bigcirc C \text { iff for every } w^{\prime} \in W, \text { if } w S w^{\prime} \text { then } w^{\prime} \models C ; \text { and } \\
& w \models * C \text { iff for every } w^{\prime} \in W, \text { if } w R w^{\prime} \text { then } w^{\prime} \models C .
\end{aligned}
$$

Note that there is no conflict between the first clause and the second two clauses, since for any complete consistent S4-theory $T$ and any formulas $C$ and $D$ in the language $L^{\square}$, we have both

$$
\begin{aligned}
& \neg C \in T \text { iff } C \notin T, \text { and } \\
& C \vee D \in T \text { iff } C \in T \text { or } D \in T .
\end{aligned}
$$

We now define the canonical birelational model $M=\langle W, S, R, V\rangle$ as follows:

$$
\begin{aligned}
& W=\{w: w \text { is a complete consistent W0/S4-theory }\} \\
& w S w^{\prime} \text { iff for every } C, \text { if } \bigcirc C \in w \text { then } C \in w^{\prime} ; \\
& w R w^{\prime} \text { iff for every } C, \text { if } * C \in w \text { then } C \in w^{\prime} ; \text { and } \\
& V(w)=\left\{C: C \in w \text { and } C \text { is in the language } L^{\square}\right\} .
\end{aligned}
$$

Note that, for any $w \in W$ and any formula $C$, we have $C \in w$ iff $w \models C$. In particular, every theorem of W0/S4 is true in every world in the canonical model.

To prove the main statement of the lemma, we will prove that $(A \vee \bigcirc B) \notin \mathrm{W} 0 / \mathrm{S} 4$ on the following three assumptions: $A$ contains no occurrences of $\bigcirc$ or $* ; A \notin \mathrm{S} 4$; and $B \notin$ W0/S4. Choose $w_{0} \in W$ such that $B \notin w_{0}$. And choose a complete consistent S4-theory $T$ such that $\neg A \in T$; this can be done since $A \notin \mathrm{S} 4$. Now define a new birelational model $M^{\prime}=\left\langle W^{\prime}, S^{\prime}, R^{\prime}, V^{\prime}\right\rangle$ as follows:

$$
\begin{aligned}
& W^{\prime}=W \cup\left\{w_{1}\right\}, \text { where } w_{1} \text { is some new world not in } W \\
& S^{\prime}=S \cup\left\{\left\langle w_{1}, w_{0}\right\rangle\right\} ; \\
& R^{\prime}=R \cup\left\{\left\langle w_{1}, w_{1}\right\rangle\right\} \cup\left\{\left\langle w_{1}, w\right\rangle: w_{0} R w\right\} ; \\
& V^{\prime}(w)=V(w), \text { for each world } w \in W ; \text { and } \\
& V^{\prime}\left(w_{1}\right)=T .
\end{aligned}
$$

We will use $\models^{\prime}$ for the validation relation in the birelational model $M^{\prime}$. Note that for every formula $C$ and every $w \in W, w \models{ }^{\prime} C$ iff $w \models C$. In particular, if $C \in$ W0/S4 then $w \models^{\prime} C$ for every $w \in W$. We also claim that if $C \in \mathrm{W} 0 / \mathrm{S} 4$ then $w_{1} \models^{\prime} C$. This is easily proved by induction on the length of proof in W0/S4. But note that $w_{1} \nvdash^{\prime}(A \vee \bigcirc B)$. So $(A \vee \bigcirc B) \notin \mathrm{W} 0 / \mathrm{S} 4$, as desired.

Proof of Lemma 47. If $A$ is of degree 0 , then the result follows from the necessitation rule and the $\mathrm{S} 4$ axioms for $\square$. Otherwise, $A \equiv(C \& D) \in \mathrm{W} 0 / \mathrm{S} 4$, where $C$ is a 
quasi-necessitive of degree $\geq 1$ and $D$ is a quasi-necessitive of degree 0 . Note that $(\neg C \equiv \bigcirc \neg \downarrow C) \in \mathrm{W} 0 / \mathrm{S} 4$. Note that $D$ is in the language $L^{\square}$ and that $(D \equiv \square D) \in \mathrm{S} 4$.

Since $(A \supset B) \in \mathrm{W} 0 / \mathrm{S} 4$, we have $((C \& D) \supset B) \in \mathrm{W} 0 / \mathrm{S} 4$. So $(\neg C \vee(D \supset B)) \in$ W0/S4. So $(\bigcirc \neg \downarrow C \vee(D \supset B)) \in \mathrm{W} 0 / \mathrm{S} 4$. So $\neg \downarrow C \in \mathrm{W} 0 / \mathrm{S} 4$ or $(D \supset B) \in \mathrm{S} 4$, by Lemma 46. So $\bigcirc \neg \downarrow C \in \mathrm{W} 0 / \mathrm{S} 4$ or $\square(D \supset B) \in \mathrm{S} 4$, by the rule of necessitation for $\bigcirc$ for W0/S4 and the rule of necessitation for $\square$ for S4. So $\bigcirc \neg \downarrow C \in \mathrm{W} 0 / \mathrm{S} 4$ or $(D \supset \square B) \in$ $\mathrm{S} 4$, by the $\mathrm{S} 4$ axioms. So $(\bigcirc \neg \downarrow C \vee(D \supset \square B)) \in \mathrm{W} 0 / \mathrm{S} 4$. So $(\neg C \vee(D \supset \square B)) \in$ W0/S4. So $((C \& D) \supset \square B) \in \mathrm{W} 0 / \mathrm{S} 4$. So $(A \supset \square B) \in \mathrm{W} 0 / \mathrm{S} 4$, as desired.

Proof of Lemma 48. Suppose that $\bigcirc A \in \mathrm{W} 0 / \mathrm{S} 4$. Then $(p \vee \bigcirc A) \in \mathrm{W} 0 / \mathrm{S} 4$ where $p$ is any propositional variable. But then $A \in \mathrm{W} 0 / \mathrm{S} 4$, since $p \notin \mathrm{S} 4$ and by Lemma 47 .

We defer the proof of Lemma 49 until after the proof of Lemma 51.

Proof of Lemma 50. We will prove the lemma on the assumption that the language is countable. First note that Lemma 49 implies that if, for every $n,\left(A \supset \bigcirc^{n} B\right) \in \mathrm{W} 0 / \mathrm{S} 4$, then $(A \supset * B) \in \mathrm{W} 0 / \mathrm{S} 4$. Now suppose that $A$ is consistent. Let $S_{1}=\{A\}$, which is a consistent $\omega$-closed set of formulas. Enumerate all the formulas as $A_{1}, \ldots, A_{n}, \ldots$. Assume that the set $S_{n}$ of formulas has been defined and is finite. If $A_{n}$ is of the form $\neg * B$ and $S_{n} \cup\left\{A_{n}\right\}$ is consistent, choose the first $m$ so that $\neg \bigcirc^{m} B$ is consistent with $S_{n} \cup\left\{A_{n}\right\}$ and let $S_{n+1}=S_{n} \cup\left\{A_{n}, \neg \bigcirc^{m} B\right\}$. Otherwise, if $A_{n}$ is not of that form but is consistent with $S_{n}$, let $S_{n+1}=S_{n} \cup\left\{A_{n}\right\}$. If $A_{n}$ is of the form $* B$ and $S_{n} \cup\left\{A_{n}\right\}$ is inconsistent, choose the first $m$ so that $\neg \bigcirc^{m} B$ is consistent with $S_{n} \cup\left\{\neg A_{n}\right\}$ and let $S_{n+1}=S_{n} \cup\left\{\neg A_{n}, \neg \bigcirc^{m} B\right\}$. Otherwise, if $A_{n}$ is not of that form but is inconsistent with $S_{n}$, let $S_{n+1}=S_{n} \cup\left\{\neg A_{n}\right\}$. Then $S=\cup_{n} S_{n}$ is a complete consistent $\omega$-closed theory with $A \in S$.

Proof of Lemma 51. $(\Rightarrow)$ This direction follows from the definition of "W0/S4-theory". $(\Leftarrow)$ Suppose $(A \supset B) \notin \mathrm{W} 0 / \mathrm{S} 4$. Then $\neg(A \& \neg B) \notin \mathrm{W} 0 / \mathrm{S} 4$. So by Lemma 50, $(A \& \neg B) \in T$ for some consistent complete $\omega$-closed W0/S4-theory $T$. So $A \in T$ and $B \notin T$. So it is not the case that for every consistent complete $\omega$-closed W0/S4-theory $T$, if $A \in T$ then $B \in T$.

Now it remains to prove Lemma 49. First, some stage setting. A finite set $\Phi$ of formulas is closed iff it is closed under subformulas and $* C \in \Phi \Rightarrow \bigcirc * C \in \Phi$, for every formula $C$. Given a closed set $\Phi$ of formulas, a $\Phi$-atom (often we just say atom) is a set $\alpha$ of signed formulas, i.e. ordered pairs of the form $+C=\langle+, C\rangle$ or $-C=\langle-, C\rangle$, where $C \in \Phi$. An atom $\alpha$ is a subatom of an atom $\beta$ iff $\alpha \subseteq \beta$. An atom $\alpha$ is $\Phi$-complete (we often just say complete) iff, for each $C \in \Phi$, either $+C \in \alpha$ or $-C \in \alpha$. An atom $\alpha$ is closed iff the set $\{C: \pm C \in \alpha\}$ is closed. Note that every $\Phi$-complete atom is closed, but not vice versa. For example, suppose that $\Phi=\{p, \bigcirc p, * p, \bigcirc * p\}$; then the atom $\alpha=\{+p,-\bigcirc p\}$ is closed but not $\Phi$-complete.

An atom is consistent iff the corresponding formula is consistent, i.e. its negation is not a theorem of $\mathrm{W} 0 / \mathrm{S} 4$. The formula corresponding to $\{+A,-B,-C\}$, for example, is $A \& \neg B \& \neg C$. We will not distinguish atoms from their corresponding formulas.

Given an atom $\alpha, \alpha_{\bigcirc}={ }_{\mathrm{df}}$ the set of signed formulas in $\alpha$ of the form $\pm \bigcirc A$; and $\alpha_{\mathrm{S} 4}={ }_{\mathrm{df}}$ the set of signed formulas in $\alpha$ containing no temporal modalities. 
Given a closed set $\Phi$ of formulas, we define some relations on $\Phi$-complete consistent $\Phi$-atoms:

$R \alpha \beta$ iff $(\alpha \& \# \beta)$ is consistent;

$S_{n} \alpha \beta$ iff $\left(\alpha \& \bigcirc^{n} \beta\right)$ is consistent;

$S \alpha \beta$ iff $S_{1} \alpha \beta$; and

$S^{n} \alpha \beta$ iff $S \alpha \alpha_{1}, \ldots, S \alpha_{n-1} \beta$, for some $\alpha_{1}, \ldots, \alpha_{n-1}$.

Before we prove Lemma 49, we first state and prove Lemma 53 to Lemma 56.

Lemma 53. Suppose that $\Phi$ is a closed set of formulas and that $\alpha$ is a closed consistent $\Phi$-atom. Then the following is a theorem of W0/S4:

$\left(\alpha_{\mathrm{S} 4} \& \alpha_{\bigcirc}\right) \supset \alpha$.

Proof. List the members of $\alpha-\left(\alpha_{S 4} \cup \alpha_{\bigcirc}\right)$ as $\pm C_{1}, \ldots, \pm C_{n}$ so that if $i<j$ then $C_{i}$ is not a subformula of $C_{j}$. Define $\alpha_{0}=\alpha$ and $\alpha_{m+1}=\alpha_{m}-\left\{ \pm C_{m+1}\right\}$, for $m=0, \ldots, n-1$. So $\alpha_{n}=\left(\alpha_{\mathrm{S} 4} \cup \alpha_{\bigcirc}\right)$. Notice also that each $\alpha_{m}$ is closed and consistent. Now it suffices to show that $\left(\alpha_{m+1} \supset \alpha_{m}\right) \in \mathrm{W} 0 / \mathrm{S} 4$, for each $m=0, \ldots, n-1$. We consider five cases.

Case 1. $\alpha_{m+1}=\alpha_{m}-\{+(D \& E)\}$. Since $\alpha_{m}$ is closed and consistent, $+D$ and $+E$ are in $\alpha_{m+1}$. So $\left(\alpha_{m+1} \supset \alpha_{m}\right)=\left(\alpha_{m+1} \supset \alpha_{m+1} \& D \& E\right) \in \mathrm{W} 0 / \mathrm{S} 4$.

Case 2. $\alpha_{m+1}=\alpha_{m}-\{-(D \& E)\}$. Since $\alpha_{m}$ is closed and consistent, either $-D$ or $-E$ is in $\alpha_{m+1}$. Assume that it is $-D$. So $\left(\alpha_{m+1} \supset \alpha_{m}\right)=\left(\alpha_{m+1} \supset \alpha_{m+1} \& \neg(D \& E)\right) \in$ W0/S4, since $(\neg D \supset \neg(D \& E)) \in \mathrm{W} 0 / \mathrm{S} 4$.

Case 3. $\alpha_{m+1}=\alpha_{m}-\{ \pm \neg D\}$. Since $\alpha_{m}$ is closed and consistent, $\mp D \in \alpha_{m+1}$. So $\left(\alpha_{m+1} \supset \alpha_{m}\right)=\left(\alpha_{m+1} \supset \alpha_{m+1} \& \pm \neg D\right) \in \mathrm{W} 0 / \mathrm{S} 4$, since $(\mp D \supset \pm \neg D) \in \mathrm{W} 0 / \mathrm{S} 4$.

Case 4. $\alpha_{m+1}=\alpha_{m}-\{+* D\}$. Since $\alpha_{m}$ is closed and consistent and since $(* D \supset$ $D \& \bigcirc * D) \in \mathrm{W} 0 / \mathrm{S} 4$, both $+D$ and $+\bigcirc * D$ are in $\alpha_{m+1}$. So $\left(\alpha_{m+1} \supset \alpha_{m}\right)=\left(\alpha_{m+1} \supset\right.$ $\left.\alpha_{m+1} \& * D\right) \in \mathrm{W} 0 / \mathrm{S} 4$, since $(D \& \bigcirc * D \supset * D) \in \mathrm{W} 0 / \mathrm{S} 4$.

Case 5. $\alpha_{m+1}=\alpha_{m}-\{-* D\}$. Since $\alpha_{m}$ is closed and consistent and since $(\neg * D \supset$ $\neg(D \& \bigcirc * D)) \in \mathrm{W} 0 / \mathrm{S} 4$, either $-D$ or $-\bigcirc * D$ is in $\alpha_{m+1}$. Let $E$ be $D$ or $\bigcirc * D$, whichever is appropriate. Then $\left(\alpha_{m+1} \supset \alpha_{m}\right)=\left(\alpha_{m+1} \supset \alpha_{m+1} \& \neg * D\right) \in \mathrm{W} 0 / \mathrm{S} 4$, since $(\neg E \supset \neg * D) \in \mathrm{W} 0 / \mathrm{S} 4$.

(Lemma 53 is inspired by [8].)

Lemma 54. Suppose that $\Phi$ is a closed set of formulas and that $S^{n}$ and $S_{n}$ are defined as above. Then for any $n \in \omega$ and any $\Phi$-complete consistent $\Phi$-atoms $\alpha$ and $\beta$, if $S^{n} \alpha \beta$ then $S_{n} \alpha \beta$.

Proof. By induction on $n$. The cases for $n=0$ and $n=1$ are obvious. For the inductive step, suppose that $S^{n+1} \alpha \beta$. Then for some atom $\delta$, we have $S \alpha \delta$ and $S^{n} \delta \beta$, so, by IH, we have $S_{n} \delta \beta$. Assume that $\neg S_{n+1} \alpha \beta$. So $\left(\alpha \supset \neg \bigcirc^{n+1} \beta\right) \in \mathrm{W} 0 / \mathrm{S} 4$. Recall that $\left(\alpha_{\mathrm{S} 4} \& \alpha \bigcirc \supset \alpha\right) \in \mathrm{W} 0 / \mathrm{S} 4$ by Lemma 53. So $\left(\alpha_{\mathrm{S} 4} \& \alpha \bigcirc \supset \neg \bigcirc^{n+1} \beta\right) \in \mathrm{W} 0 / \mathrm{S} 4$. So, 
$\left(\neg \alpha_{\mathrm{S} 4} \vee\left(\alpha \bigcirc \supset \neg \bigcirc^{n+1} \beta\right)\right) \in \mathrm{W} 0 / \mathrm{S} 4$. Since $\alpha$ is consistent, so is $\alpha_{\mathrm{S} 4}$, so $\neg \alpha_{\mathrm{S} 4} \notin \mathrm{S} 4$. So, by Lemma 46, we have

$$
\left(\alpha \bigcirc \supset \neg \bigcirc^{n+1} \beta\right) \in \mathrm{W} 0 / \mathrm{S} 4 \text {. }
$$

Now recall that $(\alpha \& \bigcirc \delta)$ is consistent. We claim that, in fact, $(\bigcirc \delta \supset \alpha \bigcirc) \in$ W0/S4. To see this, first note that $C \in \Phi$ for every signed formula $\pm \bigcirc C \in \alpha \bigcirc$, since $\alpha$ is a $\Phi$-atom. So, for every signed formula $\pm \bigcirc C \in \alpha \bigcirc$, either $+C$ or $-C$ is in $\delta$. Since $\alpha$ is consistent with $\bigcirc \delta$, we have (1) $+\bigcirc C \in \alpha_{\bigcirc}$ iff $+C \in \delta$; and (2) $-\bigcirc C \in \alpha_{\bigcirc}$ iff $-C \in \delta$. So for every signed formula $\pm \bigcirc C \in \alpha \bigcirc$, we have $(\bigcirc \delta \supset \pm \bigcirc C) \in$ W0/S4. And so $(\bigcirc \delta \supset \alpha \bigcirc) \in \mathrm{W} 0 / \mathrm{S} 4$.

So, by $(*),\left(\bigcirc \delta \supset \neg \bigcirc^{n+1} \beta\right) \in \mathrm{W} 0 / \mathrm{S} 4$. So $\bigcirc\left(\delta \supset \neg \bigcirc^{n} \beta\right) \in \mathrm{W} 0 / \mathrm{S} 4$. So $(\delta \supset$ $\left.\neg \bigcirc^{n} \beta\right) \in \mathrm{W} 0 / \mathrm{S} 4$ by Lemma 47. But this contradicts $S_{n} \delta \beta$.

Lemma 55. Suppose that $\Phi$ is a closed set of formulas and that $R$ and $S^{n}$ are defined as above. Also suppose that $\alpha$ and $\beta$ are $\Phi$-complete consistent $\Phi$-atoms. Then if $R \alpha \beta$ then $S^{n} \alpha \beta$ for some $n \in \omega$.

Proof. We adapt the third clause of the proof of Lemma 1 of [10]. Suppose that $\neg S^{n} \alpha \beta$ for every $n \in \omega$. We want to show that $\neg R \alpha \beta$. Note that $\beta \notin Y$, where $Y=\{\delta: \delta$ is a $\Phi$-complete consistent $\Phi$-atom and $S^{n} \alpha \delta$ for some $\left.n \in \omega\right\}$. We claim, for every $\Phi$-complete consistent $\Phi$-atom $\delta$,

$$
\text { if } \delta \in Y \text { then }(\delta \supset \bigcirc \vee Y) \in \mathrm{W} 0 / \mathrm{S} 4 \text {. }
$$

To see (*), suppose that $\delta \in Y$ is a $\Phi$-complete consistent $\Phi$-atom. Let $Z=\{\gamma: \gamma$ is a $\Phi$ complete consistent $\Phi$-atom and $(\delta \& \bigcirc \gamma)$ is consistent $\}$. Then $Z \subseteq Y$. So $(\vee Z \supset \vee Y) \in$ W0/S4. So $(\bigcirc \vee Z \supset \bigcirc \vee Y) \in \mathrm{W} 0 / \mathrm{S} 4$. Furthermore, $(\delta \supset \bigcirc \vee Z) \in \mathrm{W} 0 / \mathrm{S} 4$, since $Z$ contains all the $\gamma$ such that $(\delta \& \bigcirc \gamma)$ is consistent. So $(\delta \supset \bigcirc \vee Y) \in \mathrm{W} 0 / \mathrm{S} 4$, as desired.

Given $(*),(\vee Y \supset \bigcirc \vee Y) \in \mathrm{W} 0 / \mathrm{S} 4$. So, by the induction axiom, $(\vee Y \supset * \vee Y) \in$ W0/S4. Clearly $\alpha \in Y$. So $(\alpha \supset \vee Y) \in \mathrm{W} 0 / \mathrm{S} 4$. So $(\alpha \supset * \vee Y) \in \mathrm{W} 0 / \mathrm{S} 4$. We also claim that $(\vee Y \supset \neg \beta) \in \mathrm{W} 0 / \mathrm{S} 4$. The reason for this is that $\beta \notin Y$, in which case $\beta$ is inconsistent with every $\delta \in Y$. So $(* \vee Y \supset * \neg \beta) \in \mathrm{W} 0 / \mathrm{S} 4$. So $(\alpha \supset * \neg \beta) \in \mathrm{W} 0 / \mathrm{S} 4$. So $\neg(\alpha \& \# \beta) \in \mathrm{W} 0 / \mathrm{S} 4$. So $\neg R \alpha \beta$, as desired.

Lemma 56. Suppose that $\Phi$ is a closed set of formulas and that $\alpha$ and $\beta$ are complete consistent $\Phi$-atoms. Then if $(\alpha \& \# \beta)$ is consistent, then $\left(\alpha \& \bigcirc^{n} \beta\right)$ is consistent for some $n \in \omega$.

Proof. This is an immediate corollary to Lemmas 54 and 55.

Proof of Lemma 49. Suppose that $(A \& \# B)$ is consistent. Let $\Phi$ be the smallest closed set of formulas such that $(A \& \# B) \in \Phi$. Let $\Phi=\left\{A_{1}, \ldots, A_{m}\right\}$ where the $A_{i}$ are all distinct and $A_{1}=(A \& \# B)$. Define $\alpha_{1}=\left\{+A_{1}\right\}$ and for each $n=2, \ldots, m$, define $\alpha_{n}=\alpha_{n-1} \cup\left\{+A_{n}\right\}$ if $A_{n}$ is consistent with $\alpha_{n-1}$, and $\alpha_{n}=\alpha_{n-1} \cup\left\{-A_{n}\right\}$ otherwise. And let $\alpha=\alpha_{m}$. Then $\alpha$ is a complete consistent $\Phi$-atom such that $+(A \& \# B) \in \alpha$. So $(\alpha \& \# B)$ is consistent. Also note that $+A \in \alpha$. 
Let $\Phi=\left\{B_{1}, \ldots, B_{m}\right\}$ where the $B_{i}$ are all distinct and $B_{1}=B$. Define $\beta_{1}=\left\{+B_{1}\right\}$ and for each $n=2, \ldots, m$, define $\beta_{n}=\beta_{n-1} \cup\left\{+B_{n}\right\}$ if $\left(\alpha \& \#\left(\beta_{n-1} \& B\right)\right)$ is consistent, and $\beta_{n}=\beta_{n-1} \cup\left\{-B_{n}\right\}$ otherwise. And let $\beta=\beta_{m}$. Then $\beta$ is a complete consistent $\Phi$-atom such that $+B \in \beta$ and $(\alpha \& \# \beta)$ is consistent. So by Lemma 56, $\left(\alpha \& \bigcirc^{n} \beta\right)$ is consistent for some $n \in \omega$. But then $\left(A \& \bigcirc^{n} B\right)$ is consistent since $+A \in \alpha$ and $+B \in \beta$.

\section{References}

[1] M. Aiello, J. van Benthem, G. Bezhanishvili, Reasoning about space: the modal way, Journal of Logic and Computation 13 (2003) 889-920.

[2] P. Alexandrov, Diskrete Räume, Matematicheskii Sbornik 2 (1937) 501-518.

[3] S. Artemov, J. Davoren, A. Nerode, Modal logics and topological semantics for hybrid systems, Technical Report MSI 97-05, Cornell University, June, 1997, available at http://www.cs.gc.cuny.edu/ $\sim$ sartemov/.

[4] N. Bourbaki, General Topology, Part 1, Hermann, Paris, Addison-Wesley, 1966.

[5] J. Brown, Ergodic Theory and Topological Dynamics, Academic Press, New York, 1976.

[6] J. Davoren, Modal logics for continuous dynamics, Ph.D. Thesis, Cornell University, 1998.

[7] H. Furstenberg, Recurrence in Ergodic Theory and Combinatorial Number Theory, Princeton University Press, Princeton, 1981.

[8] R. Goldblatt, Logics of Time and Computation, 2nd edition, Center for the Study of Language and Information Lecture Notes, no. 7, Stanford University Press, Stanford, 1992.

[9] B. Konev, R. Kontchakov, D. Tishovsky, F. Wolter, M. Zakharyaschev, On dynamic topological and metric logics, 2004 (manuscript).

[10] D. Kozen, R. Parikh, An elementary proof of the completeness of PDL, Theoretical Computer Science 14 (1981) 113-118.

[11] P. Kremer, Temporal logic over S4: an axiomatizable fragment of dynamic topological logic, Bulletin of Symbolic Logic 3 (1997) 375-376.

[12] P. Kremer, G. Mints, Dynamic topological logic, Bulletin of Symbolic Logic 3 (1997) 371-372.

[13] P. Kremer, G. Mints, V. Rybakov, Axiomatizing the next-interior fragment of dynamic topological logic, Bulletin of Symbolic Logic 3 (1997) 376-377.

[14] S. Kripke, Semantical analysis of modal logic I, normal propositional calculi, Zeitschrift für Mathematische Logik und Grundlagen der Mathematik 9 (1963) 67-96.

[15] F. Kröger, Temporal Logics of Programs, Springer-Verlag, Berlin, 1985.

[16] J.C.C. McKinsey, A solution of the decision problem for the Lewis systems S2 and S4, with an application to topology, The Journal of Symbolic Logic 6 (1941) 117-134.

[17] J.C.C. McKinsey, A. Tarski, The algebra of topology, Annals of Mathematics 45 (1944) 141-191.

[18] G. Mints, T. Zhang, A proof of topological completeness for S4 in $(0,1)$ (forthcoming).

[19] A.N. Prior, Past, Present and Future, Clarendon Press, Oxford, 1967.

[20] H. Rasiowa, R. Sikorski, The Mathematics of Metamathematics, Państowowe Wydawnictwo Naukowe, Warsaw, 1963.

[21] K. Segerberg, Discrete linear future time without axioms, Studia Logica 35 (1976) 273-278.

[22] K. Segerberg, Von Wright's logic of time, in: Logitjeskij Vyvod, Nauka, Moscow, 1979 (in Russian).

[23] K. Segerberg, Von Wright's tense logic, in: P.A. Schilpp, L.E. Hahn (Eds.), The Philosophy of Georg Henrick von Wright, Open Court, La Salle, 1989, pp. 603-635.

[24] S. Slavnov, Two counterexamples in the logic of dynamic topological systems, Technical Report TR2003015, Cornell University, 2003.

[25] T.-C. Tang, Algebraic postulates and a geometric interpretation for the Lewis calculus of strict implication, Bulletin of the American Mathematical Society 44 (1938) 737-744.

[26] G.H. von Wright, And next, Acta Philosophica Fennica facs 18 (1965) 293-304.

[27] G.H. von Wright, Always, Theoria 34 (1968) 208-221.

[28] P. Walters, An Introduction to Ergodic Theory, Springer-Verlag, Berlin, 1982. 